\title{
Social touch alters newborn monkey behavior
}

Elizabeth A. Simpson ${ }^{1 *}$, Sarah E. Maylott ${ }^{1}$, Roberto J. Lazo ${ }^{1}$, Kyla A. Leonard ${ }^{1,2}$, Stefano S. K. Kaburư ${ }^{3}$, Stephen J. Suomi ${ }^{4}$, Annika Paukner ${ }^{5}$, Pier F. Ferrari ${ }^{6}$

${ }^{1}$ Department of Psychology, University of Miami, Coral Gables, Florida, USA

${ }^{2}$ Department of Psychology, Northern Illinois University, DeKalb, Illinois, USA

${ }^{3}$ Department of Biomedical Science \& Physiology, Faculty of Science \& Engineering, University of Wolverhampton, Wolverhampton, England

${ }^{4}$ Eunice Kennedy Shriver National Institute of Child Health and Human Development, National Institutes of Health, Poolesville, Maryland, USA

${ }^{5}$ Department of Psychology, Nottingham Trent University, Nottingham, England

${ }^{6}$ Institut des Sciences, Cognitives-Marc Jeannerod, Université Claude Bernard, Lyon, France

*Corresponding author: simpsone@miami.edu; 5665 Ponce De Leon Boulevard, Coral Gables, Florida 33146, USA

\section{Accepted for publication in Infant Behavior and Development on 8-27-19.}

Author Contributions: E.A.S., P.F.F., A.P., and S.S.K.K. designed the studies. E.A.S. and S.S.K.K. collected the data. E.A.S., S.E.M, K.A.L., and R.J.L. analyzed, visualized, and interpreted the data. E.A.S. wrote the paper. All authors edited and approved the final manuscript.

Acknowledgements: We thank the research and animal care staff in the Laboratory of Comparative Ethology for their support of this project. Thanks to Katalin Gothard for providing the monkey video stimulus.

Funding: This work was supported by the Division of Intramural Research, Eunice Kennedy Shriver National Institute of Child Health and Human Development, National Institutes of Health (AP); National Institutes of Health Grant Number: P01HD064653 (PFF); and National Science Foundation CAREER Award 1653737 (EAS).

Data Statement: Data will be made available upon request.

Competing Interests Statement: The authors have no competing interests to declare.

\section{Highlights}

- Few studies have explored the role of touch in infants' psychological development.

- We investigated the acute effects of social (CT-targeted) touch in newborn monkeys.

- Infants' environments were experimentally controlled, offering unique insight.

- During touch, infants exhibited fewer stress-related behaviors.

- Eye tracking revealed that touch influenced some aspects of infants' attention. 


\begin{abstract}
In humans, infants respond positively to slow, gentle stroking-processed by C-tactile (CT) nerve fibers-by showing reductions in stress and increases in eye contact, smiling, and positive vocalizations. More frequent maternal touch is linked to greater activity and connectivity strength in social brain regions, and increases children's attention to and learning of faces. It has been theorized that touch may prime children for social interactions and set them on a path towards healthy social cognitive development. However, less is known about the effects of touch on young infants' psychological development, especially in the newborn period, a highly sensitive period of transition with rapid growth in sensory and social processing. It remains untested whether newborns can distinguish CT-targeted touch from other types of touch, or whether there are benefits of touch for newborns' social, emotional, or cognitive development. In the present study, we experimentally investigated the acute effects of touch in newborn monkeys, a common model for human social development. Rhesus macaques (Macaca mulatta), like humans, are highly social, have complex mother-infant interactions with frequent body contact for the first weeks of life, making them an excellent model of infant sociality. Infant monkeys in the present study were reared in a neonatal nursery, enabling control over their early environment, including all caregiver interactions. One-week-old macaque infants $(N=27)$ participated in three 5-minute counter-balanced caregiver interactions, all with mutual gaze: stroking head and shoulders (CT-targeted touch), stroking palms of hands and soles of feet (Non-CT touch), or no stroking (No-touch). Immediately following the interaction, infants watched social and nonsocial videos and picture arrays including faces and objects, while we tracked their visual attention with remote eye tracking. We found that, during the caregiver interactions, infants behaved differently while being touched compared to the no-touch condition, irrespective of the body part touched. Most notably, in both touch conditions, infants exhibited fewer stress-related behaviors-self-scratching, locomotion, and contact time with a comfort object-compared to when they were not touched. Following CTtargeted touch, infants were faster to orient to the picture arrays compared to the other interaction conditions, suggesting CT-targeted touch may activate or prime infants' attentional orienting system. In the No-touch condition infants attended longer to the nonsocial compared to the social video, possibly reflecting a baseline preference for nonsocial stimuli. In contrast, in both touch conditions, infants' looked equally to the social and nonsocial videos, suggesting that touch may influence the types of visual stimuli that hold infants' attention. Collectively, our results reveal that newborn macaques responded positively to touch, and touch appeared to influence some aspects of their subsequent attention, although we found limited evidence that these effects are mediated by CT fibers. These findings suggest that newborn touch may broadly support infants' psychological development, and may have early evolutionary roots, shared across primates. This study illustrates the unique insight offered by nonhuman primates for exploring early infant social touch, revealing that touch may positively affect emotional and attentional development as early as the newborn period.
\end{abstract}

Keywords: neonate, social attention, tactile, CT fibers, infant behavior, face perception, sociability 
Touch is one of the first senses to develop prenatally (Bradley \& Mistretta, 1975; Marx \& Nagy, 2015) and one of the earliest forms of parent-infant communication (Field, 2001; Hertenstein, Verkamp, Kerestes, \& Holmes, 2006). After birth, mothers remain in close proximity to their infants and actively touch them; these behaviors are instinctive, evolutionarily conserved, and widely shared across mammals (Feldman, 2011, 2015). Infants, in turn, seek and develop attachments to providers of contact comfort (Harlow \& Zimmermann, 1959), engaging in bi-directional, mutually regulated touch interactions (Mantis, Stack, Ng, Serbin, \& Schwartzman, 2014). Touch is critical for infants' growth and for the development of healthy immune, endocrine, and nervous systems (Feldman, 2011; Field, 2010; Underdown, Barlow, \& Stewart-Brown, 2010). However, there are fewer empirical investigations exploring the potential role of touch in supporting infants' social, emotional, or cognitive development (Bales et al., 2018; Field, 2019; Gliga, Farroni, \& Cascio, 2019). In the present study, we hypothesized that the effects of touch may not be limited to infants' physical health, but may also extend to infants' psychological functioning, reducing stress and promoting infant social behavior during and immediately following touch interactions with caregivers. We predicted that sensitivity to social touch may already be present in newborns, and theorized that it may serve to regulate infant emotions and help infants establish social connections in the first weeks after birth.

\section{CT-Targeted "Social" Touch}

Caregivers use many types of touch, which serve a variety of functions. For example, caregivers often use touch to attract infants' attention, to play, to show affection, and to reduce infants' distress (Jean \& Stack, 2009; Jean, Stack, \& Fogel, 2009). One specific type of touch appears to play a central role in social interactions: social or affective touch (Field, 2019). Parents often use this type of touch, gently caressing infants with affectionate, slow, gentle strokes (Ferber, Feldman, \& Makhoul, 2008). One type of social touch that may play a central role in infants' early development is touch that activates C-tactile (CT) afferent fibers, a type of unmyelinated peripheral nerve fiber, which respond preferentially to medium velocity soft brushing touch (Ackerley et al., 2014; Croy et al., 2016; Field, 2019; Gordon et al., 2013; McGlone et al., 2014). CT fibers are particularly good at conveying affective or social information. For example, studies in adults report that CT fibers project to brain regions involved in affective processing such the insular cortex and other regions critical for social cognition, including the posterior superior temporal sulcus, medial prefrontal cortex, amygdala, and ventrolateral prefrontal cortex (Bennett et al., 2014; Björnsdotter \& Olausson, 2011; Gordon et al., 2013). Unlike other touch receptors, CT fibers are located all over the body, especially in places with hair-concentrated on top of the head, upper torso, arms and thighs-but not glabrous (hairless) skin, e.g., lips, palms of the hands, and soles of the feet (Olausson et al., 2002). Little is known, however, about the role of CT-targeted touch in supporting infants' development (Bales et al., 2018; Field, 2019; Gliga et al., 2019).

\section{Touch Regulates Stress}

Postnatal touch may play an important role in regulating infants' emotions and stress (Morrison, 2016). In stressful contexts, it may be adaptive for infants to seek out social contact (Taylor, 2006; Taylor et al., 2000), and touch may serve an important regulatory role. For example, in preterm newborns, gentle touch reduces infants' motor activity and behavioral distress, and decreases arousal (Harrison, Williams, Berbaum, Stem, \& Leeper, 2000). At 5 to 6 months old, when placed in a stressful situation (i.e., face-to-face still-face task), infants display fewer stress behaviors while being touched by a caregiver, crying less and smiling more, and display fewer physiological indicators of stress, including higher vagal tone and lower cortisol levels (Feldman, Singer, \& Zagoory, 2010; Stack \& Muir, 1992). Similarly, 6-month-old infants seek contact comfort when confronted with a novel, potentially frightening object (Crockenberg \& Leerkes, 2004). Touch functions to connect caregivers and infants, even when other communicative channels, such as face-to-face interaction, are disrupted, lessening 
infants' stress levels (Jean \& Stack, 2009). In both adults (Mayo, Lindé, Olausson, \& Heilig, 2018; Pawling, Cannon, McGlone, \& Walker 2017) and 1- to 9-month-old infants (Aguirre, Couderc, Epinat-Duclos, \& Mascaro, 2019; Fairhurst, Loken, \& Grossman, 2014; Peláez-Nogueras et al., 1996), social touch reduces heart rate and increases smiling, suggesting it may have similar effects across the lifespan, reducing arousal and increasing positive affect.

\section{Touch Facilitates Sociality}

In addition to reducing stress and negative arousal, touch may also promote social interactions and facilitate social perception. Correlational evidence suggests that rates of maternal touch may be associated with children's neurobehavioral, social, and cognitive growth. For example, in preterm and full-term infants, rates of spontaneous maternal touch and holding were associated with alertness during social interactions and were positively associated with infant cognitive skills and quality of mother-infant interaction at 6 and 12 months (Feldman \& Eidelman, 2003a; Korja et al., 2018). Similarly, 4- to 6-year-olds whose mothers naturally touched them more during a free-play interaction were subsequently more socially attentive, looking longer at faces compared to children whose mothers touched them less (Reece, Ebstein, Cheng, $\mathrm{Ng}$, Schirmer, 2016). Further, in 5-year-old children, rates of spontaneous maternal touch during free-play were positively associated with activity and connectivity in social brain regions, including the superior temporal sulcus and dorso-medial prefrontal cortex (Brauer, Xiao, Poulain, Friederici, \& Schirmer, 2016). Together, these studies offer preliminary support for the proposal that touch may affect infants' psychological development, potentially promoting infants' social responsiveness and engagement.

However, it is difficult to determine causality from these correlational studies. Infants and caregivers mutually influence one another, so it is impossible to determine the extent to which the patterns observed are due to some characteristics of the child that is affecting parental touch, and/or whether there are differences in parents' touch, which are affecting children's responses. Touch also cooccurs with a variety of other caregiving behaviors, including face-to-face interactions with mutual gaze, parental vocalizations, feeding, play, and other interactions, which may interactively impact development. Experimental studies are necessary to specifically isolate the effects of touch from the milieu of other caregiving behaviors (Dettmer et al., 2016a; Simpson et al., 2019). To date, there are only a handful of experimental studies examining the psychological effects of touch in infants. For example, in small-for-gestational-age infants (i.e., full-term, low birth weight), those who received a tactile stimulation intervention went on to have higher levels of social interactions at 6 and 12 months of age, compared to infants who did not receive the intervention (Watt, 1990). One study found that preterm infants who received massage therapy, compared to a no-massage control group, engaged in more reciprocal interactions with their mothers at 3 months of age (Ferber et al., 2005). Another study found that 4-month-old infants who received gentle stroking by parents while observing faces were more likely to learn facial identities compared to a no-touch condition (Della Longa, Gliga, \& Garroni, 2019). A recent study compared parent-infant interactions during walks while children were either pushed in strollers or carried in backpacks, in which they were in physical contact with parents (Mireault, Rainville, \& Laughlin, 2018). They found that, during babywearing, 7- to 11-month-olds had more dyadic conversations with parents, including more infant vocalizations and more infant-initiated interactions, compared to when infants were in strollers. Facilitating touch through babywearing may encourage infants' language and communication development. These studies suggest that touch may facilitate infants' social learning and engagement.

\section{The Newborn Period: An Animal Model to Explore a Unique Stage in Development}

We theorize that, in the newborn period, touch may be important for establishing the first patterns of mother-infant exchanges, as one of many sensory-motor modalities involved in the mutual 
regulation of affect. Newborns are responsive to social stimuli (Schultz, Klin, \& Jones, 2018), and in the first weeks after birth, infants' expressiveness increases (Murray et al., 2016). Furthermore, studies in primates suggest that newborns' social behaviors are malleable (Dettmer et al., 2016a; Simpson et al., 2014, 2016a; Vanderwert et al., 2015), reflecting a rapid period of changes in early development, and indicating a particularly sensitive period for interventions. However, it remains untested whether newborns can distinguish CT-targeted touch from other types of touch and stimulation (Jönsson et al., 2018), whether there are any effects of touch on newborns' social, emotional, or cognitive development, and if so, what type of touch may optimally support infants' development. Primate models can offer insights into the underlying biological influences on complex psychological phenomenon, such as infant-caregiver interactions, and shed light on the evolution of these traits (Gerson et al., 2016; Maestripieri, 1999; Maestripieri \& Roney, 2006; Reeb-Sutherland, 2018).

Macaque monkey newborns are an ideal population in which to explore social touch in infancy. Rhesus macaques (Macaca mulatta) are a highly social species with strong mother-infant bonds characterized by complex face-to-face interactions in the first weeks of life (Dettmer et al., 2016a, 2016b; Ferrari, Paukner, lonica, \& Suomi, 2009). Nonhuman primates allow a level of experimental control that is impossible to achieve in studies of humans, enabling us to isolate the effects of social touch outside the milieu of other caregiving behaviors (Drury, Sánchez, \& Gonzalez, 2015; Gerson, Simpson, \& Paukner, 2016). Despite the central role of maternal touch in early infant development, the mechanisms remain largely unexplored due to the difficulty of disentangling touch from all of the other caregiver provisions (e.g., feeding, mutual gaze, infant-directed speech; Fairhurst et al., 2014; Hofer, 2006; Shibata et al., 2012; Underdown et al., 2010; Weaver et al., 2004). In macaques we can systematically assess each of these factors experimentally (Simpson et al., 2019). In addition, newborn macaques are visually precocious (Ordy, Latanick, Samorajski, \& Massopust, 1964) and we can accurately assess their visual attention via remote eye tracking (Paukner, Simpson, Ferrari, Mrozek, \& Suomi, 2014), a method that is not yet feasible in human newborns, but which has unique translational value for the diagnosis and treatment of neurodevelopmental disorders (Bauman \& Schumann, 2018; Parr et al., 2016; Ryan et al., 2019).

\section{Current Study}

In the present study, we explored the effects of social touch on infant monkey behavior with macaque infants reared by humans in a neonatal nursery. This environment offers the unique advantage of eliminating any maternal interindividual differences in touch or other early environmental differences, which cannot be controlled in human studies. We hypothesized that social (CT-targeted) touch would reduce infants' stress-related behaviors, and increase both their positive social behaviors, and their attentiveness to the environment, during and immediately following social touch. We predicted that stroking infants' head and shoulders (CT-targeted touch) would elevate infants' visual attention (e.g., more rapid look latencies and longer look durations), especially to socially relevant stimuli relative to equally engaging (novel, colorful, dynamic) nonsocial stimuli. In contrast, when infants were stroked on their hands and feet (non-CT) or received no stroking at all, we predicted infants would be less socially attentive.

\section{Methods}

The Eunice Kennedy Shriver National Institute of Child Human Development Animal Care and Use Committee approved all procedures. The study was conducted in accordance with the Guide for the Care and Use of Laboratory Animals, APA ethical standards in the treatment of animals, and complied with the Animal Welfare Act.

\section{Subjects}


We tested 27 healthy infant rhesus macaques, 16 females and 11 males, between 7 and 15 days of age ( $M=10.9$ days, $S D=1.9)$, born in two cohorts, including $n=16$ ( 9 females) born in 2015, and $n=$ 11 ( 7 females) born in 2016. Infants were separated from their mothers on the day of birth and reared in a nursery for unrelated studies. This nursery-rearing environment enabled a high degree of standardization in infants' social interactions (Simpson et al., 2016a). In the first two weeks after birth, infants were individually housed in warmed incubators $(51 \times 38 \times 43 \mathrm{~cm})$ that contained an inanimate, fleece-covered surrogate mother, loose pieces of fleece fabric, and various plastic and plush toys. Infants were fed Similac infant formula. Infants could see and hear, but not physically contact, other infants of similar age. Human caretakers were present for 13 hours daily and interacted with infants every 2 hours for feeding and cleaning. While not species-typical, these infants did have consistent, positive social contact from human caretakers, who interacted with infants in ways similar to macaque mothers, providing consistent and regular mutual gaze, stroking, and carrying (for details, see: Simpson et al., 2016a). Accordingly, long-term studies have shown that nursery-reared infant macaques display a social development that is similar to infants reared by their mothers (Champoux, Metz, \& Suomi, 1991). In addition, the inanimate soft surrogates provide continuous contact comfort when the human caretakers were not present. Some of these infants also participated in other unrelated behavioral studies, including tests of their social, sensory, and motor development. We avoided testing infants on days in which they experienced other testing, to avoid fatigue effects. If an infant was fussy or sleepy, testing was attempted again on the following day, to ensure all infants contributed usable data.

\section{Materials}

Video pair stimuli. Infants were presented with two side-by-side 10-second videos: an object moving (i.e., plastic bag blowing in the wind) and an adult female rhesus macaque lipsmacking, an affiliate facial gesture in this species (see Video 1 for sample clip). After viewing the video-pair once for 10 seconds, the left-right video positions were switched for a second 10-second trial (Figure 1a). We chose these stimuli because they have high ecological validity, reflective of the real world in which infants have numerous dynamic stimuli competing for their attention (Pierce et al., 2016).

[Figure 1 here]

Picture array stimuli. Infants viewed up to four trials, each consisting of an 8-item circular array of pictures, including two direct-gaze adult rhesus macaque monkey faces and six nonsocial distractors (e.g., plants, animals, toys, bottles; Figure 2a). In two arrays the own-species faces were upright, and in the other two the own-species faces were inverted. Upright and inverted face locations and distractors were counter-balanced across infants. In total, each infant saw 32 unique static images, including eight different conspecifics. The same stimuli were shown across each of the three test days.

[Figure 2 here]

Eye tracking apparatus. We recorded infants' eye movements using corneal reflection through a Tobii TX300 eye tracker with a $58.4 \mathrm{~cm}$ monitor with integrated eye tracking technology, with the screen resolution set to $1280 \times 720$, and a sampling rate of 60 Hertz. Infants were tested in a room where sunlight was blocked, and illumination of 250 lux was achieved by one overhead light (approximately 4 feet behind the infant) and one additional light to the right of the infant. We collected and summarized the eye tracking data with Tobii Studio software (Tobii Technology, Danderyd, Sweden).

\section{Procedure}


Touch manipulation sessions. Infants were awake and fed prior to testing. In a repeated measures design, each infant participated in three manipulation conditions, carried out across three separate days (one per day). The order of the conditions was randomized for each infant. One experimenter was the model (Experimenter 1), who made eye contact with and touched the infants. A second experimenter (Experimenter 2) videotaped the infant during the interaction. Each infant received a 5-minute manipulation, which took place while the infants were in their home incubators. Incubator tops were wire mesh, allowing the infant to see out. Experimenter 1 positioned herself just outside of the infant's cage, at eye-level, and attempted to make and maintain eye contact with the infant while maintaining a neutral facial expression, while performing one of three forms of tactile stimulation: (a) stroking the infant's head and shoulders; (b) stroking the infant's hands and feet; or (c) not touching the infant (gaze only). Testing occurred once per day, always at the same time of day and with the same experimenters.

Strokes were carried out to maximize activation of CT fibers (Croy et al., 2016; Vallbo, Olausson, \& Wessberg, 1999): they were slow, with a velocity of approximately $3-5 \mathrm{~cm} / \mathrm{sec}$, and light/gentle (i.e., low-force) stroking movements, and Experimenter 1 applied these to the infant with one hand, alternating between two body parts-either head and shoulders, or hands and feet-with five strokes each. For example, Experimenter 1 would apply a stroke on the infant's head followed by a stroke on the infant's shoulder, repeated and alternating left-right sides. While previous studies of CT-targeted touch most commonly have focused on the forearm (for a review, see: Field, 2019), we instead chose to stroke the head and neck regions, specifically, because they are rich in CT fibers (Olausson et al., 2002), and are areas of the body where human and macaque mothers naturally touch their infants (Ferrari et al., 2009; Moreno, Posada, \& Goldyn, 2006). Human adults report CT-targeted touch to be particularly pleasurable in these locations (Panagiotopoulou, Filippetti, Gentsch, \& Fotopoulou, 2018), and these are intimate regions of the body, where human adults report that they would only be comfortable with someone close to them, such as their mothers, touching them (Suvilehto, Glerean, Dunbar, Hari, \& Nummenmaa, 2015).

Strokes to the hands and feet were directed at the palms of the hands and the soles of the feet, parts of the body that are glabrous (hairless) and appear to lack tactile $\mathrm{C}$ afferent fibers (Liljencrantz \& Olausson, 2014; McGlone et al., 2012; Vallbo et al., 1999). If one of the areas was inaccessible, then only the accessible area was stroked. For example, if the infant was standing so the foot soles had full contact with the floor and could not be reached, then we would stroke the accessible part (in this case, the tops of the feet). Similarly, if the infant was clutching something, preventing us from stroking the infant's palm, we would instead stroke the back of the hand. In the no touch control condition, Experimenter 1 gazed at, but did not touch, the infant during the 5-minute session. Nitrile rubber medical gloves were worn for all touch manipulations due to health and safety protocols of working with nonhuman primates. All manipulation sessions were videotaped for later analysis.

Eye tracking. Immediately following the touch manipulation, the infant was swaddled by Experimenter 1 and carried to an adjoining room for two eye tracking tasks. Experimenter 1 held the infant during eye tracking while Experimenter 2 controlled the eye tracking computer, to present stimuli and record infant looking. Earlier the same day each infant was calibrated using a 5-point calibration to Tobii Studio's pre-set locations, therefore allowing an efficient transition to eye tracking, which started within two minutes of the end of the touch manipulation. Experimenter 1 held the infant approximately $60 \mathrm{~cm}$ from the screen. A central cartoon and music attracted the infant's attention to the center of the screen, at which time Experimenter 2 pressed a key to start the first trial. Two 10-second long video trials were shown first (Figure 1a). Then, infants viewed four trials of image arrays (Figure 2a) in random order, each shown until the infant accumulated 10 seconds of cumulative looking, monitored by Experimenter 2 via live-tracking. The eye tracking took approximately 5 minutes. 


\section{Data Analysis}

Behavior during caregiver interactions. To assess reactions to touch, we coded the 5-minute caregiver interaction videos for 14 common infant monkey behaviors (Simpson et al., 2016b, 2019; see Table 1). Four behaviors-vocalizations, self-suck, self-clasp, sleep-occurred rarely or not at all, so were excluded from subsequent analyses. We were particularly interested in infant behaviors related to arousal and stress-e.g., self-scratching, contact with the surrogate (comfort object), locomotion-and social interest, e.g., lipsmacking facial gestures, attention to the caregiver's face, and proximity to the caregiver. Two independent coders rated each behavior. We assessed inter-rater reliability in $59 \%$ of the videos ( $n=48$ ), which revealed high levels of agreement (average ICC $=.84, r s>.90, p s<.001$ ). We carried out a one-way analysis of variance (ANOVA) on each infant behavior, exploring the between subjects independent variable of condition (No Touch, Hand/Foot Touch, Head/Shoulder Touch). We also examined whether there were specific behavioral profiles patterns associated with the different touch manipulations by assessing whether the infants' behaviors were correlated, and we combined multiple behaviors into a smaller number of composite measures. We then carried out one-way ANOVAs on each composite measure exploring Condition. Given the large number of statistical tests, we used Bonferroni corrections (detailed below).

Attention after caregiver interactions: Eye tracking. We drew areas of interest (AOI) around each video (Figure $1 \mathrm{~b}$ ) and image (Figure $2 \mathrm{~b}$ ) and extracted data using the Tobii filter in Tobii Studio. We measured attention capture (look latency) and attention holding (look duration), which are related and distinct aspects of visual processing (Cohen, 1972), and have been previously used in newborn monkeys (Simpson et al., 2017).

We first measured infants' attention to the videos. Two equally sized AOls, 640 (width) $\times 380$ (height) pixels each, contained the two videos (social and nonsocial). First, we tested for overall effects on attentiveness (not specific to stimulus type) more broadly, to determine if there may be some general attentional change. To assess this we carried out two one-way ANOVAs exploring condition, one on look latency (time from the start of the trial until the first fixation to each video), and one on look duration (amount of time looking). For the look latency data, we trimmed scores that fell more than 2 SD from the mean, which resulted in the exclusion of 6 scores out of 155 total ( $4 \%$ of the data). We next tested whether there may be a shift in the types of stimuli (relative interest in social compared to nonsocial) that infants attended to. For this analysis, we carried out a one-way ANOVA on the proportion of time looking to the social (time looking to the social video divided by the time looking to both videos) to test for effects of condition. We also conducted one-sample t-tests, within each condition, to determine if infants' rates of looking differed from chance (looking half the time to each video).

We next measured infants' attention to the picture arrays. Eight equally sized AOIs, sized 200 (width) $\times 200$ (height) pixels each, contained each individual picture of the arrays. Mirroring our approach with the analysis of the video stimuli, our eye tracking data analysis for the picture stimuli included two types of tests: First, we tested for overall effects on attentiveness (not specific to stimulus type), to determine if there may be broad, general attentional change. To assess this we carried out two one-way ANOVAs exploring condition, one on look latency (time from the start of the trial to the first fixation to each image), and one on look duration (amount of time looking). For the look latency data, we trimmed scores that fell more than 2 SD from the mean within each condition, which resulted in the exclusion of 36 scores out of 546 total ( $6 \%$ of the data). We next tested whether there may be a shift in the types of stimuli (relative interest in social compared to nonsocial photos) that infants attended to. For this analysis we carried out two $2 \times 3$ mixed design ANOVAs, one on look latency and one on look duration, each exploring the within-subjects variable of picture type (face, non-face) and the between subjects variable of condition. 


\section{Behavior During Caregiver Interactions}

\section{Results and Discussion}

We first explored infant behaviors during the touch manipulation sessions by carrying out a series of 9 one-way ANOVAs on each of our dependent measures (Figure S1). To account for inflated Type I Error rates, we used Bonferroni adjusted $p$-values $(.05 / 9=.005)$. This revealed only two statistically significant effects (see Supplemental Materials for details on the non-statistically significant effects).

Locomotion. The first statistically significant effect we found was on locomotion. Locomotion may be an indicator of arousal or stress in infant monkeys (Spinelli et al., 2012). We found an effect of condition on time spent in locomotion, $F(2,52)=6.34, p=.003, \eta_{p}{ }^{2}=.196$ (Figure S1c). Infants moved more in the No Touch $(M=40.32$ seconds, $S D=31.01)$ compared to either the Head/Shoulder Touch $(M$ $=23.86$ seconds, $S D=23.67), t(26)=2.94, p=.007, d=0.58$, or Hand/Foot Touch conditions $(M=22.77$ seconds, $S D=29.49), t(26)=3.11, p=.004, d=0.61$. There was no difference in locomotion between the Head/Shoulder and Hand/Foot conditions, $t(26)=.21, p=.84$. Infants who were not touched may have been experiencing higher levels of anxiety.

Exploration. Our second statistically significant effect was on exploration. We found an effect of condition on time spent exploring, $F(2,52)=9.62, p<.001, \eta_{\mathrm{p}}{ }^{2}=.270$ (Figure S1d). Infants explored more in the No Touch $(M=25.89$ seconds, $S D=36.88)$ compared to either the Head/Shoulder $(M=2.82$ seconds, $S D=9.66), t(26)=3.04, p=.005, d=0.60$, or the Hand/Foot conditions $(M=2.42$ seconds, $S D=$ $5.28), t(26)=3.32, p=.003, d=0.65$. There was no difference in exploration between the two touch conditions, $t(26)=0.18, p=.86$. At first, this finding seems at odds with previous research findings that social touch-giving nursery-reared macaque newborns additional daily handling-increases exploration of novel people, objects, and environments (Simpson et al., 2019). However, the present study focused on infants' behavior in their familiar home environment with a familiar caregiver, which may elicit different types of behaviors compared to less familiar stimuli. It could also be that chronic and acute touch elicit different effects, with chronic touch reducing infants' baseline stress/arousal levels, decreasing fear and increasing exploration of novel stimuli, while acute touch may temporarily relax infants, making them move around less and giving them something to focus on.

Internalizing Stress. To examine if there were specific behavioral profile patterns associated with the different touch manipulations, we explored which specific behaviors were related to one another and therefore could potentially be combined (Table S1). This revealed positive correlations among behaviors in three domains: (1) behaviors associated with internalizing stress (i.e., stress reactions directed towards self)-self-scratching, contact with surrogate (comfort object), and locomotion; (2) behaviors associated with externalizing stress (i.e., stress reactions directed towards others) - vocalizations, spasms, and yawns; and (3) behaviors associated with social interestlipsmacking, visually attending to the caregiver, and time in close proximity to the caregiver. We therefore standardized (converted to z-scores) and combined (averaged together) these behaviors to create three composite measures. We then carried out repeated measures ANOVAs on each composite measure to explore the effects of condition. For the internalizing stress composite we found a main effect of condition, $F(2,52)=12.28, p<.001, \eta_{p}{ }^{2}=.321$ (Figure 3 ). Infants exhibited a higher internalizing stress score when in the No Touch condition $(M=.37, S D=.72)$ compared to the Head/Shoulder Touch condition $(M=.17, S D=.62)$ and the Hand/Foot Touch condition $(M=.21, S D=.45), t(26)=3.74, p=$ $.001, d=.72$, and $t(26)=4.67, p<.001, d=.90$, respectively. There were no differences between the two touch conditions, $t(26)=.31, p=.76$. For the social interest composite, there was no effect of condition (No Touch: $M=.18, S D=.84$; Hand/Foot: $M=-.16, S D=.53$; Head/Shoulder: $M=-.02, S D=.61), F(2,52$ ) $=2.00, p=.15$. For the externalizing stress composite, there was no effects of condition (No Touch: $M=-$ $.08, S D=.85 ;$ Hand/Foot: $M=.01, S D=.49 ;$ Head/Shoulder: $M=.08, S D=.66), F(2,52)=.66, p=.52$. 
Together, these results suggest that, even within a brief (5-minute) window of interaction, infants may have been more stressed when they were not being touched.

[Figure 3 here]

\section{Attention After Caregiver Interactions: Eye Tracking}

Attention to videos overall. We first analyzed infants' attention to the videos. We assessed whether infants' overall rates of attentiveness to the videos varied across conditions with repeated measures ANOVAs on overall look latency and duration. We detected no significant differences in infants' look latency across the No Touch $(M=2.21 \mathrm{sec}, S D=1.31)$, Hand/Foot $(M=2.57 \mathrm{sec}, S D=1.24)$, and Head/Shoulder $(M=2.09 \mathrm{sec}, S D=1.65)$ conditions, $F(2,50)=.43, p=.65$. We detected no significant differences in infants' overall attentiveness (look duration to both videos) across the No Touch $(M=7.49 \mathrm{sec}, S D=5.07)$, Hand/Foot $(M=8.23 \mathrm{sec}, S D=4.38)$, and Head/Shoulder $(M=7.77 \mathrm{sec}$, $S D=4.51)$ conditions, $F(2,52)=.26, p=.78$. Together, these results suggest that touching did not significantly alter infants' overall attention to dynamic visual stimuli.

Attention to social vs. non-social videos. We were also interested in whether touching influenced the specific types of items that attracted infants' attention, namely, their relative interest in the social and nonsocial videos. To explore this question, we assessed infants' relative looking to the social and nonsocial videos with a one-way ANOVA on the proportion of time looking to the social video. We found no significant effect of condition, $F(2,52)=2.03, p=.14$ (Figure 4a). This suggests that infants' proportion of looking to the social video did not vary substantially across our three conditions. However, we were also interested in exploring whether, within each condition, infants' attention differed from chance (equal looking to both the social and nonsocial videos), so we conducted three one-sample $t$ tests (one for each condition), which revealed that infants in the No Touch condition looked significantly less at the social video $(M=3.56 \mathrm{sec}, S D=.63)$ relative to the nonsocial video $(M=3.94 \mathrm{sec}, S D=.55)$, attending to the nonsocial video most (59.36\%) of the time, $t(26)=2.14, p=.042, d=.41$. The other two conditions-Hand/Foot and Head/Shoulder-did not differ from chance, $t(26)=.20, p=.84$, and $t(26)=$ $.45, p=.66$, respectively. We found no other significant differences in infants' look latencies or look durations to the social and nonsocial videos for any condition, $p s>.05$ (Figure S2). Together, these findings suggest that infants with limited social experience may have had a baseline preference for the nonsocial video, but that being touched-in either touch condition-increased their relative interest in the social video.

[Figure 4 here]

Attention to pictures overall. We next measured infants' attention to the picture arrays. We assessed whether infants' overall rates of attentiveness to the picture arrays varied across condition with two repeated measures ANOVAs for the dependent measures of look latency and look duration. We detected a significant main effect of condition for infants' look latency, $F(2,52)=4.87, p=.012, \eta_{p}{ }^{2}=$ .158 , in which infants were faster to look in the Head/Shoulder Touch condition $(M=10.02 \mathrm{sec}, S D=$ 5.17) compared to the No Touch condition ( $M=16.11 \mathrm{sec}, S D=10.19), t(26)=2.04, p=.005, d=.59$ (Figure 4b). Infants also showed a non-significant trend of being faster to look in the Head/Shoulder Touch condition compared to the Hand/Foot Touch condition $(M=13.24 \mathrm{sec}, S D=6.29), t(26)=2.02, p$ $=.054, d=.39$. We detected no significant differences in infants' overall attentiveness (look duration to all images) across conditions, $F(2,52)=.171, p=.843$ (see Figure $S 3$ ). These results suggest that the social touch (i.e., Head/Shoulder touch) condition specifically may have a general influence on attention capture, that helps infants to efficiently detect and orient to visual stimuli in their environment, but that touch condition did not seem to influence attention holding. These findings are consistent with the 
proposal that attention capture and attention holding are distinct attentional mechanisms in infancy (Cohen, 1972), and suggest that there may be something about social touch that may prime infants for interacting with the world around them.

Attention to social vs. non-social pictures. We were also interested in whether touching influenced the specific types of items that attracted infants' attention, in particular, their relative interest in the social and nonsocial pictures. To explore this question, we assessed infants' relative looking to the upright conspecific face photos relative to the other (nonsocial) photos with repeated measures ANOVAs looking at picture type (face, non-face) and condition (No Touch, Hand/Foot, Head/Shoulder), for both look latency and look duration. We found no significant effects of condition for look latency, $p s>.05$ (Figure S3; top graph). For look duration, we found only a main effect of picture type in which infants spent more time looking at the non-face images $(M=.15 \mathrm{sec}, S D=.01)$ compared to the face images $(M=.13 \mathrm{sec}, S D=.03), F(2,34)=3.73, p=.034, \eta_{\mathrm{p}}{ }^{2}=.18$ (Figure S3; bottom graph). This effect likely reflects the fact that the non-face images-particularly the toys, butterflies, and flowers-had greater low-level salience (e.g., brightness, contrast) compared to the faces, so infants attended to them longer, consistent with previous reports in human infants (Kwon, Setoodehnia, Baek, Luck, \& Oakes, 2016). There were no other significant effects, $p s>.05$. In sum, the types of images that captured and held infants' attention did not seem to vary as a function of touch. That is, we found no evidence that touch increased newborn monkey social orienting to face photos, as reported in older children (Reece et al., 2016).

\section{General Discussion}

The goal of the present study was to explore whether slow, gentle stroking (CT-targeted touch), compared to non-CT stroking, or no touch, influenced neonatal monkeys' behaviors during and immediately following a 5-minute social interaction with a familiar caregiver. We found that newborn monkeys, who share with humans many other features of their early social behavior and physiology, behaved differently when being touched, showing reductions in stress-related behaviors, regardless of the body part touched (CT-targeted or non-CT touch). They also exhibited subsequent changes in attention immediately following touch, displaying overall faster visual orienting and revealing that there may be small increases in the attention-holding of dynamic social stimuli. While it remains to be tested whether these effects will also appear in humans, the present study establishes that it is social touch itself, and not other aspects of the social caregiving milieu, that influences social attention. Although the importance of touch for social bonding across primate species is well known (Dunbar, 2010), the current study highlights the utility of the macaque infant model to shed light on the evolutionary and neurobiological mechanisms of social touch in infancy. The long-term effects of social touch remain to be explored; however, the present findings suggest that acute touch may alter newborn monkeys' behavior. These short-term effects, if accumulated over time, may have notable consequences on infant psychological development and well-being (Bales et al., 2018; Field, 2019; Gliga et al., 2019).

\section{Infant Behavior During Touch}

In line with our predictions, we found that infants responded differently during mutual gaze with a caregiver while being touched, compared to mutual gaze only (without touch). Specifically, we found that while infants were being touched they seemed more relaxed and less stressed, showing reductions in exploratory behaviors and locomotion, consistent with decreased arousal (Simpson et al., 2019), less contact time with a surrogate comfort-object, a self-soothing behavior (Van Horen \& Mussweiler, 2014), and less self-scratching, an indicator of anxiety (Kaburu et al., 2016). These findings are consistent with the proposal that touch may function to decrease arousal, sooth infants, and reduce stress-related behaviors (Morrison, 2016). Previous studies in human-reared monkey infants reported that touch interactions in the first month of life-i.e., additional handling by caregivers beyond routine 
care-were positively associated with infants' social working memory, and rates of facial gesturing to a novel social partner, and exploration of novel objects and environments between 2 weeks and 3 months of age, suggesting infants who received more handling may have had more advanced social cognitive capacities and were less fearful and less socially anxious compared to infants who received less handling (Simpson et al., 2019). These previous findings are consistent with our findings in the present study that, when infants were touched, compared to when they were not, they exhibited fewer stress related behaviors. Another possibility is that infants were simply more distracted in the touch conditions when they were receiving more stimulation, compared to the no-touch conditions, which may have drawn their attention away from any stressful emotions they were experiencing. Indeed, in humans, infants in the neonatal intensive care unit show reductions in stress when positive distractions, such when as nature, arts, and music, are introduced (Shepley, 2006). It is also possible that these infants had elevated stress levels due to being reared in a neonatal nursery, and touch may have reduced this stress, bringing their levels down to be more species-typical. Nonetheless, the touch was effective, and regardless of the specific mechanisms, it seems that infants behaved more positively when being touched. While the present study only explored acute (short-term) touch, there are likely both short- and longer-term impacts of receiving healthy levels of social touch.

We did not, however, find any behavioral differences in infants during touch that was specific to the CT-targeted touch. That is, infants behaved in similar ways when they were touched using CTtargeted and non-CT stroking. We theorized that sensitivity to social touch emerges early in development, based on reports in human infants that, much like adults, 2-month-olds display stronger neural responses to CT-targeted touch, compared to non-CT (faster stroking) touch, with distinct patterns of activation in the insular cortex and temporal lobe (Jönsson et al., 2018; also see Aguirre et al., 2019; Miguel, Lisboa, Gonçalves, \& Sampaio, 2019; Pirazzoli, Lloyd-Fox, Braukmann, Johnson, \& Gliga, 2019). Therefore, we expected infants to display behavioral differences in response to CT-touch as well.

While it is possible that infants at this age do not yet show CT-targeted specific effects, we think this is unlikely. There are a number of reasons why we may have failed to find evidence that monkey newborns display preferences for CT-targeted touch. First, we only measured infants' behaviors in one specific context, i.e., short-term touch during mutual gaze in familiar home environment with familiar caregiver. It is possible that a more stressful environment would have elicited larger effects, given the stress-reducing power of touch demonstrated in human infants (Feldman et al., 2010; Harrison et al., 2000; Stack et al., 1992). Further, we were limited in only measuring infants' behavioral responses; their physiological-based changes (e.g., heart rate) may have been more sensitive for showing differences. In addition, the social touch we used in the present study was not contingent upon infants' behaviors (e.g., sensitive to an infant's emotional state), which may have limited its effectiveness (Crucianelli et al., 2019). Indeed, 7- to 30-day-old macaques exhibit more affiliative and prosocial behaviors during a contingent social interaction with a caregiver compared to a non-contingent interaction (Sclafani, Paukner, Suomi, \& Ferrari, 2015), suggesting they are already remarkably socially sensitive in the first weeks after birth and recognize when a social partner is not responding in a contingent way. Ideally, caregivers' stimulation would be tailored to each infant's individual preferences and needs. However, our goal was to have high experimental control, so our stimulation was consistent and did not vary as a function of the infants' reactions to being touched. Future studies could explore touch in a more naturalistic, contingent context, in a wider variety of environments.

Another component of the social interaction task in the present study that may have inadvertently affected infants' behavior is the mutual-gaze with the neutral expression. In human infants, in contrast, this unresponsive, neutral face elicits stressful behaviors, including increased fussiness and crying, even in newborns (Nagy et al., 2017), who prefer faces that are communicative (Cecchini, Baroni, Di Vito, Piccolo, \& Lai, 2011). Interestingly, when social touch accompanies the still- 
face, this reduces infants' stressful reactions: they cry less, smile more, and display fewer physiological indicators of stress (Bigelow \& Power, 2012; Feldman et al., 2010; Jean \& Stack, 2009; Mantis et al., 2014; Stack \& Muir, 1992). We are unaware of any studies testing whether nonhuman primate infants show similar stress reactions to a caregiver's still-face. In macaques, although staring behavior (i.e., direct gaze) is commonly displayed in an aggressive context to threaten conspecifics (Maestripieri, 1997), such threat expressions are not typically directed to newborns, to our knowledge. Though unlikely, it is possible that in the present study we may have inadvertently increased newborns' stress using this approach. This might explain why we found, during the gaze-only no-touch condition, infants seemed more stressed, locomoting more, and showing increases in time with their surrogate comfort objects, more self-scratching, and more attempts to distract themselves through exploring other things in their environment. However, in infant monkeys of this age, mutual gaze is not associated with aggression, but instead with affiliation (Paukner et al., 2014; Ferrari et al. 2009). In fact, adults often engage in long bouts of mutual gaze with infants, in a positive/affiliative context, sometimes (but not always) accompanied by grooming, kisses, exaggerated facial and vocal expressions (motherese), head bobbing, and other positive affiliative exchanges (Ferrari et al., 2009; Dettmer et al., 2016). Therefore, we think it is unlikely that these infants, who were in a familiar environment with a familiar caretaker, would find this direct eye-contact to be threatening or aversive. If anything, we think it likely signaled to them a precursor to a social interaction, and infants' movements and increased activity were a consequence of them anticipating further social contact (i.e., excited to be held).

\section{Infant Attention Immediately Following Touch}

We did not detect CT-specific effects during touch; however, we did find CT-specific effects immediately following touch, in infants' subsequent visual attention, measured with eye tracking. We found that CT-targeted touch primed newborns' attention, increasing attention capture generally and resulting in faster orienting to pictures. This effect appeared to be broad, impacting orienting to all pictures in a general way, not specific to any particular type of picture. Heightened attention capture in infants may reflect the anticipation of, or preparation for, an interaction (Galazka \& Nyström, 2016). In addition, vision and touch have early sensory interactions, and studies in adults reveal that stimulation in one sensory modality can influence efficiency in the other (Orchard-Mills, Alais, \& Van der Burg, 2013). The findings in the present study open up the possibility that the attentional effects of social touch may not be specific to social stimuli only, and additional studies with nonsocial control stimuli are necessary to test this possibility. To date, most studies of social touch explored children's attentiveness to social but not nonsocial stimuli. For example, in human infants, 4-month-olds appear better at learning facial identities when that learning is accompanied by social touch compared to non-CT conditions (Della Longa et al., 2019); however, as these authors acknowledge, it is possible that social touch may be enhancing learning in a more general way, not specific to faces.

In addition to these overall attention capture effects, we also found that, in both touch conditions, infants shifted their attentional focus from preferring a nonsocial video (in the no-touch condition), to spending more equal time attending to a nonsocial and social video, suggesting there may be a small social priming effect. However, we did not find statistically significant differences across our conditions, suggesting this apparent effect may not be robust, or could even be an artifact of our small sample size. This result, therefore, should be interpreted with caution, and should be verified through replication studies. If it is a true effect, this finding - that both types of touch shifted infants' attentional focus towards dynamic social stimuli-is consistent with previous observational studies in humans. For example, in 4- to 6-year-old children, more maternal touch is associated with greater social attentiveness, i.e., more attentive to photos of faces compared to houses, and greater brain resting activity and connectivity in areas associated with mentalizing, suggesting enhanced social cognitive skills (Brauer et al., 2016; Reece et al., 2016). In adults, simply observing others being touched increases social 
attentiveness to faces (Schirmer, Ng, \& Ebstein, 2018), and gentle human (CT-targeted) touch, compared to non-CT touch, increases pupillary dilation while observing faces, associated with attentional orienting (Ellingsen et al., 2014). The present findings suggest that, even in newborns, social touch may similarly increase infants' attraction to social stimuli.

What mechanisms might underlie the association between touch and social attention? Higher levels of oxytocin-a neuropeptide involved in parental, romantic, and filial bonds (Feldman, 2012)-are associated with positive social behaviors. For example, in humans, newborns' oxytocin levels in cerebrospinal fluid are positively associated with their sociability (Clark et al., 2013) and children's salivary oxytocin levels are positively associated with their social attention (Nishizato, Fujisawa, Kosaka, \& Tomoda, 2017). Similarly, in macaque newborns, administering oxytocin increases infants' social interest (Simpson et al., 2014b; Simpson et al., 2017). Touch appears to trigger the release of oxytocin (Morrison, 2016). For example, parent-child interactions increase children's salivary oxytocin levels, particularly among children with low baseline levels (Feldman, Golan, Hirschler-Guttenberg, OstfeldEtzion, \& Zagoory-Sharon, 2014). Endogenous oxytocin can now be measured in saliva in early infancy (Fujiwara et al., 2019), so future research can test whether social touch may increase infants' oxytocin levels, moderating their subsequent social attention.

Even though we may not detect strong population-level effects across our touch and no-touch conditions, it is possible that some infants may be showing touch-related benefits. For example, perhaps infants who engage in more mutual gaze during touch (but not during caregiver interactions without touch) are subsequently more socially attentive after caregiver interactions, showing stronger attentional effects. Indeed, previous studies in newborn macaques have reported that mutual gazeinvolving touch, eye-contact, and contingent facial gesturing-when they occur daily as part of infant macaques' early newborn environment, appear to increase infants' attention to a social relative to a nonsocial video stimulus at 1 month of age (Dettmer et al., 2016a). In the present study, we unfortunately did not have a large enough sample of infants to test for interindividual differences.

Another limitation of the present study is that these infants did not experience a species-typical early environment. Human caretakers, rather than adults of their own species, reared these infants, which probably influenced their early social development (Simpson, Suomi, \& Paukner, 2016). Future studies should test infant macaques raised in more species-typical environments to determine the generalizability of our findings. For example, recent advancements in infant nonhuman primate eye tracking methods now enable researchers to capture infant nonhuman primate attention without disrupting their natural social groups. For example, researchers can remotely track infant macaque gaze patterns while they remain clinging to their biological mothers (Muschinski, Feczko, Brooks, Collantes, Heitz, \& Parr, 2016), by placing them into a box with a peep-hole for viewing stimuli (Ryan et al., 2019), or, when they are young ( 7 to 30 days after birth), swaddling them and having a human caretaker hold them (Paukner, Slonecker, Murphy, Wooddell, \& Dettmer, 2018). Future studies using one or more of these approaches, can help to disentangle the effects of specific types of early social experiences on infant sensitivity to social touch, to better uncover the translational value of various nonhuman primate infant populations as models for different aspects of human development, including infants at risk of developmental challenges (Capitanio, 2017; McCowan et al., 2016; Sclafani et al., 2016).

\section{Conclusions}

The newborn period is a distinct, sensitive, and especially stressful time in development, as infants adjust to the transition from the in utero to the postnatal environment (Nagy, 2011). Given the stress-reducing power of touch, newborns may benefit from this contact. The present study revealed that newborn primates can distinguish CT-targeted touch from other types of touch, and that touch may positively impact newborns' emotional and attentional development. Our findings add to a growing body of work in humans suggesting neonatal social touch may broadly support not only infants' healthy 
physical development, but also their psychological development (Anisfeld et al., 1990; Korja et al., 2018; Mireault et al., 2018; Watt, 1990). The effects of social touch may be far-reaching, potentially impacting attention capture and holding earlier in development than previously reported. Longitudinal experimental studies should explore a wider variety of infant outcomes, especially those related to infants' information processing, memory, and attention, in both social and nonsocial contexts. Animal studies - which enable a level of experimental control that is not possible in humans - will be particularly helpful for uncovering the mechanisms that underlie these processes (Dunbar, 2010) and to test their malleability across the lifespan (Fleming et al., 1999; Simpson et al., 2019). Such studies will enable better support for human infants, especially those at risk of developmental disorders or delays (Mantis, Meruci, Stack, \& Field, 2019), ultimately improving their health and well-being. 


\section{References}

Aguirre, M., Couderc, A., Epinat-Duclos, J., \& Mascaro, O. (2019). Infants discriminate the source of social touch at stroking speeds eliciting maximal firing rates in CT-fibers. Developmental Cognitive Neuroscience, 36, 100639. doi: 10.1016/j.den.2019.100639

Anisfeld, E., Casper, V., Nozyce, M., \& Cunningham, N. (1990). Does infant carrying promote attachment? An experimental study of the effects of increased physical contact on the development of attachment. Child Development, 61(5), 1617-1627. doi: 10.1111/j.14678624.1990.tb02888.x

Bales, K. L., Witczak, L. R., Simmons, T. C., Savidge, L. E., Rothwell, E. S., Rogers, F. D., Manning, R. A., Heise, M. J., Englund, M., \& del Razo, R. A. (2018). Social touch during development: Long-term effects on brain and behavior. Neuroscience \& Biobehavioral Reviews, 95, 202-219. doi: 10.1016/j.neubiorev.2018.09.019

Bauman, M. D., \& Schumann, C. M. (2018). Advances in nonhuman primate models of autism: Integrating neuroscience and behavior. Experimental Neurology, 299, 252-265. doi: 10.1016/j.expneurol.2017.07.021

Bennett, R. H., Bolling, D. Z., Anderson, L. C., Pelphrey, K. A., \& Kaiser, M. D. (2014). fNIRS detects temporal lobe response to affective touch. Social Cognitive and Affective Neuroscience, 9(4), 470-476. doi: 10.1093/scan/nst008

Bigelow, A. E., \& Power, M. (2012). The effect of mother-infant skin-to-skin contact on infants' response to the Still Face Task from newborn to three months of age. Infant Behavior and Development, 35(2), 240-251. doi: 10.1016/j.infbeh.2011.12.008

Björnsdotter, M., \& Olausson, H. (2011). Vicarious responses to social touch in posterior insular cortex are tuned to pleasant caressing speeds. The Journal of Neuroscience, 31(26), 9554-9562. doi: 10.1523/JNEUROSCI.0397-11.2011

Bradley, R. M., \& Mistretta, C. M. (1975). Fetal sensory receptors. Physiological Reviews, 55(3), 352-382.

Brauer, J., Xiao, Y., Poulain, T., Friederici, A. D., \& Schirmer, A. (2016). Frequency of maternal touch predicts resting activity and connectivity of the developing social brain. Cerebral Cortex, 26(8), 3544-3552. doi: 10.1093/cercor/bhw137

Capitanio, J. P. (2017). Naturally occurring nonhuman primate models of psychosocial processes. Institute for Laboratory Animal Research Journal, 58(2), 226-234. doi: 10.1093/ilar/ilx012

Cecchini, M., Baroni, E., Di Vito, C., Piccolo, F., \& Lai, C. (2011). Newborn preference for a new face vs. a previously seen communicative or motionless face. Infant Behavior and Development, 34(3), 424-433. doi: 10.1016/j.infbeh.2011.04.002

Champoux, M., Metz, B., \& Suomi, S. J. (1991). Behavior of nursery/peer-reared and mother-reared rhesus monkeys from birth through 2 years of age. Primates, 32(4), 509-514. doi: 10.1007/BF02381941

Clark, C. L., John, N. S., Pasca, A. M., Hyde, S. A., Hornbeak, K., Abramova, M., ... \& Penn, A. A. (2013). Neonatal CSF oxytocin levels are associated with parent report of infant soothability and sociability. Psychoneuroendocrinology, 38(7), 1208-1212. doi: 10.1016/j.psyneuen.2012.10.017

Cohen, L. B. (1972). Attention-getting and attention-holding processes of infant visual preferences. Child Development, 43, 869-879. doi: 10.2307/1127638

Crockenberg, S. C., \& Leerkes, E. M. (2004). Infant and maternal behaviors regulate infant reactivity to novelty at 6 months. Developmental Psychology, 40(6), 1123-1132. doi: 10.1037/00121649.40.6.1123

Croy, I., Luong, A., Triscoli, C., Hofmann, E., Olausson, H., \& Sailer, U. (2016). Interpersonal stroking touch is targeted to $C$ tactile afferent activation. Behavioural Brain Research, 297, 37-40. doi: 10.1016/j.bbr.2015.09.038 
Crucianelli, L., Wheatley, L., Filippetti, M. L., Jenkinson, P. M., Kirk, E., \& Fotopoulou, A. K. (2019). The mindedness of maternal touch: An investigation of maternal mind-mindedness and motherinfant touch interactions. Developmental Cognitive Neuroscience, 35, 47-56. doi: 10.1016/j.dcn.2018.01.010

Della Longa, L., Gliga, T., \& Farroni, T. (2019). Tune to touch: Affective touch enhances learning of face identity in 4-month-old infants. Developmental Cognitive Neuroscience, 35, 42-46. doi: 10.1016/j.den.2017.11.002Get

Dettmer, A. M., Kaburu, S. S., Simpson, E. A., Paukner, A., Sclafani, V., Byers, K. L., ... \& Ferrari, P. F. (2016a). Neonatal face-to-face interactions promote later social behaviour in infant rhesus monkeys. Nature Communications, 7, 11940. doi: 10.1038/ncomms11940

Dettmer, A. M., Kaburu, S. S., Byers, K. L., Murphy, A. M., Soneson, E., Wooddell, L. J., \& Suomi, S. J. (2016b). First-time rhesus monkey mothers, and mothers of sons, preferentially engage in faceto-face interactions with their infants. American Journal of Primatology, 78(2), 238-246. doi: https://doi.org/10.1002/ajp.22503

Drury, S. S., Sánchez, M. M., \& Gonzalez, A. (2015). When mothering goes awry: Challenges and opportunities for utilizing evidence across rodent, nonhuman primate and human studies to better define the biological consequences of negative early caregiving. Hormones and Behavior, 77, 182-192. doi: 10.1016/j.yhbeh.2015.10.007

Dunbar, R. I. (2010). The social role of touch in humans and primates: behavioural function and neurobiological mechanisms. Neuroscience \& Biobehavioral Reviews, 34(2), 260-268. doi: 10.1016/j.neubiorev.2008.07.001

Edwards, R. C., \& Hans, S. L. (2015). Infant risk factors associated with internalizing, externalizing, and co-occurring behavior problems in young children. Developmental Psychology, 51(4), 489-499. doi: $10.1037 / a 0038800$

Ellingsen, D. M., Wessberg, J., Chelnokova, O., Olausson, H., Laeng, B., \& Leknes, S. (2014). In touch with your emotions: oxytocin and touch change social impressions while others' facial expressions can alter touch. Psychoneuroendocrinology, 39, 11-20. doi: 10.1016/j.psyneuen.2013.09.017

Fairhurst, M. T., Löken, L., \& Grossmann, T. (2014). Physiological and behavioral responses reveal 9month-old infants' sensitivity to pleasant touch. Psychological Science, 25(5), 1124-1131. doi: $10.1177 / 0956797614527114$

Feldman, R. (2011). Maternal touch and the developing infant. Handbook of Touch: Neuroscience, Behavioral, and Health Perspectives, M. J. Hertenstein \& S. J. Weiss (Eds). Springer: New York. pp. 373-407.

Feldman, R. (2012). Oxytocin and social affiliation in humans. Hormones and Behavior, 61(3), 380-391.

Feldman, R. (2015). The adaptive human parental brain: implications for children's social development. Trends in Neurosciences, 38(6), 387-399. doi:10.1016/j.tins.2015.04.004

Feldman, R., \& Eidelman, A. I. (2003a). Direct and indirect effects of breast milk on the neurobehavioral and cognitive development of premature infants. Developmental Psychobiology, 43(2), 109-119. doi: 10.1002/dev.10126

Feldman, R., \& Eidelman, A. I. (2003b). Skin-to-skin contact (Kangaroo Care) accelerates autonomic and neurobehavioural maturation in preterm infants. Developmental Medicine \& Child Neurology, 45(4), 274-281. doi: 10.1111/j.1469-8749.2003.tb00343.x

Feldman, R., Golan, O., Hirschler-Guttenberg, Y., Ostfeld-Etzion, S., \& Zagoory-Sharon, O. (2014). Parentchild interaction and oxytocin production in pre-schoolers with autism spectrum disorder. The British Journal of Psychiatry, 205(2), 107-112. doi: 10.1192/bjp.bp.113.137513

Feldman, R., Singer, M., \& Zagoory, O. (2010). Touch attenuates infants' physiological reactivity to stress. Developmental Science, 13(2), 271-278. doi: 10.1111/j.1467-7687.2009.00890.x

Ferrari, P. F., Paukner, A., Ionica, C., \& Suomi, S. J. (2009). Reciprocal face-to-face communication 
between rhesus macaque mothers and their newborn infants. Current Biology, 19(20), 17681772. doi: 10.1016/j.cub.2009.08.055

Ferrari, P. F., Visalberghi, E., Paukner, A., Fogassi, L., Ruggiero, A., \& Suomi, S. J. (2006). Neonatal imitation in rhesus macaques. PLoS Biology, 4(9), e302. doi: 10.1371/journal.pbio.0040302

Ferber, S. G., Feldman, R., Kohelet, D., Kuint, J., Dollberg, S., Arbel, E., \& Weller, A. (2005). Massage therapy facilitates mother-infant interaction in premature infants. Infant Behavior and Development, 28(1), 74-81. doi: 10.1016/j.infbeh.2004.07.004

Ferber, S. G., Feldman, R., \& Makhoul, I. R. (2008). The development of maternal touch across the first year of life. Early Human Development, 84(6), 363-370. doi: 10.1016/j.earlhumdev.2007.09.019

Festante, F., Vanderwert, R. E., Sclafani, V., Paukner, A., Simpson, E. A., Suomi, S. J., ... \& Ferrari, P. F. (2018). EEG beta desynchronization during hand goal-directed action observation in newborn monkeys and its relation to the emergence of hand motor skills. Developmental Cognitive Neuroscience, 30, 142-149. doi: 10.1016/j.dcn.2018.02.010

Field, T. (2019). Social touch, CT touch and massage therapy: A narrative review. Developmental Review, 51, 123-145. doi: 10.1016/j.dr.2019.01.002

Field, T. (2001). Touch. Cambridge, MA: MIT Press.

Field, T. (2010). Touch for socioemotional and physical well-being: A review. Developmental Review, 30(4), 367-383. doi: 10.1016/j.dr.2011.01.001

Fleming, A. S., O'Day, D. H., \& Kraemer, G. W. (1999). Neurobiology of mother-infant interactions: experience and central nervous system plasticity across development and generations. Neuroscience \& Biobehavioral Reviews, 23(5), 673-685. doi: 10.1016/S0149-7634(99)00011-1

Fujiwara, T., Weisman, O., Ochi, M., Shirai, K., Matsumoto, K., Noguchi, E., \& Feldman, R. (2019). Genetic and peripheral markers of the oxytocin system and parental care jointly support the crossgenerational transmission of bonding across three generations. Psychoneuroendocrinology, 102, 172-181. doi: 10.1016/j.psyneuen.2018.12.004

Galazka, M., \& Nyström, P. (2016). Visual attention to dynamic spatial relations in infants and adults. Infancy, 21(1), 90-103. doi: 10.1111/infa.12091

Gerson, S., Simpson, E. A., \& Paukner, A. (2016). Drivers of social cognitive development in human and non-human primate infants. In J. Sommerville, \& J. Decety (Eds.), Frontiers in Developmental Science Series: Social Cognition. Psychology Press, Taylor and Francis Group. pp. 98-128.

Gliga, T., Farroni, T., \& Cascio, C. J. (2019). Social touch: A new vista for developmental cognitive neuroscience? Developmental Cognitive Neuroscience, 35, 1-4. doi: 10.1016/j.dcn.2018.05.006

Gordon, I., Voos, A. C., Bennett, R. H., Bolling, D. Z., Pelphrey, K. A., \& Kaiser, M. D. (2013). Brain mechanisms for processing affective touch. Human Brain Mapping, 34(4), 914-922. doi: $10.1002 / \mathrm{hbm} .21480$

Harlow, H. F., \& Zimmermann, R. R. (1959). Affectional responses in the infant monkey. Science, 130, 421-432. doi: 10.1126/science.130.3373.421

Harrison, L. L., Williams, A. K., Berbaum, M. L., Stem, J. T., \& Leeper, J. (2000). Physiologic and behavioral effects of gentle human touch on preterm infants. Research in Nursing \& Health, 23(6), 435-446. doi: 10.1002/1098-240X(200012)23:6<435::AID-NUR3>3.0.CO;2-P

Hertenstein, M. J., Verkamp, J. M., Kerestes, A. M., \& Holmes, R. M. (2006). The communicative functions of touch in humans, nonhuman primates, and rats: A review and synthesis of the empirical research. Genetic, social, and general psychology monographs, 132(1), 5-94.

Hofer, M. A. (2006). Psychobiological roots of early attachment. Current Directions in Psychological Science, 15(2), 84-88. doi: 10.1111/j.0963-7214.2006.00412.x

Jean, A. D., \& Stack, D. M. (2009). Functions of maternal touch and infants' affect during face-to-face 
interactions: New directions for the still-face. Infant Behavior and Development, 32(1), 123-128. doi: 10.1016/j.infbeh.2008.09.008

Jean, A. D., Stack, D. M., \& Fogel, A. (2009). A longitudinal investigation of maternal touching across the first 6 months of life: Age and context effects. Infant Behavior and Development, 32(3), 344-349. doi: 10.1016/j.infbeh.2009.04.005

Jönsson, E. H., Kotilahti, K., Heiskala, J., Wasling, H. B., Olausson, H., Croy, I., .. \& Karlsson, L. (2018). Affective and non-affective touch evoke differential brain responses in 2-month-old infants. Neuroimage, 169, 162-171. doi: 10.1016/j.neuroimage.2017.12.024

Kaburu, S. S., Paukner, A., Simpson, E. A., Suomi, S. J., \& Ferrari, P. F. (2016). Neonatal imitation predicts infant rhesus macaque (Macaca mulatta) social and anxiety-related behaviours at one year. Scientific Reports, 6, 34997. doi: 10.1038/srep34997

Korja, R., Maunu, J., Kirjavainen, J., Savonlahti, E., Haataja, L., Lapinleimu, H., ... \& PIPARI Study Group. (2008). Mother-infant interaction is influenced by the amount of holding in preterm infants. Early Human Development, 84(4), 257-267. doi: 10.1016/j.earlhumdev.2007.06.006

Kwon, M. K., Setoodehnia, M., Baek, J., Luck, S. J., \& Oakes, L. M. (2016). The development of visual search in infancy: Attention to faces versus salience. Developmental Psychology, 52(4), 537-555. doi: $10.1037 /$ dev0000080

Liljencrantz, J., \& Olausson, H. (2014). Tactile C fibers and their contributions to pleasant sensations and to tactile allodynia. Frontiers in Behavioral Neuroscience, 8, 37. doi: 10.3389/fnbeh.2014.00037

Maestripieri, D. (1997). Gestural communication in macaques: Usage and meaning of nonvocal signals. Evolution of communication, 1(2), 193-222. doi: 10.1075/eoc.1.2.03mae

Maestripieri, D. (1999). The biology of human parenting: Insights from nonhuman primates. Neuroscience \& Biobehavioral Reviews, 23(3), 411-422. doi: 10.1016/S0149-7634(98)00042-6

Maestripieri, D., \& Roney, J. R. (2006). Evolutionary developmental psychology: Contributions from comparative research with nonhuman primates. Developmental Review, 26(2), 120-137. doi: 10.1016/j.dr.2006.02.006

Mantis, I., Mercuri, M., Stack, D. M., \& Field, T. M. (2019). Depressed and non-depressed mothers' touching during social interactions with their infants. Developmental Cognitive Neuroscience, 35, 57-65. doi: 10.1016/j.den.2018.01.005

Mantis, I., Stack, D. M., Ng, L., Serbin, L. A., \& Schwartzman, A. E. (2014). Mutual touch during motherinfant face-to-face still-face interactions: Influences of interaction period and infant birth status. Infant Behavior and Development, 37(3), 258-267. doi: 10.1016/j.infbeh.2014.04.005

Marx, V., \& Nagy, E. (2015). Fetal behavioural responses to maternal voice and touch. PloS ONE, 10(6), e0129118. doi: 10.1371/journal.pone.0129118

Mayo, L. M., Lindé, J., Olausson, H., \& Heilig, M. (2018). Putting a good face on touch: Facial expression reflects the affective valence of caress-like touch across modalities. Biological Psychology, 137, 83-90. doi: 10.1016/j.biopsycho.2018.07.001

McGlone, F., Olausson, H., Boyle, J. A., Jones-Gotman, M., Dancer, C., Guest, S., \& Essick, G. (2012). Touching and feeling: differences in pleasant touch processing between glabrous and hairy skin in humans. European Journal of Neuroscience, 35(11), 1782-1788. doi: 10.1111/j.14609568.2012.08092.x

Mesman, J., van IJzendoorn, M. H., \& Bakermans-Kranenburg, M. J. (2009). The many faces of the StillFace Paradigm: A review and meta-analysis. Developmental Review, 29(2), 120-162. doi: 10.1016/j.dr.2009.02.001

McGlone, F., Wessberg, J., \& Olausson, H. (2014). Discriminative and affective touch: sensing and feeling. Neuron, 82(4), 737-755. doi: 10.1016/j.neuron.2014.05.001

Miguel, H. O., Lisboa, I. C., Gonçalves, Ó. F., \& Sampaio, A. (2019). Brain mechanisms for processing 
discriminative and affective touch in 7-month-old infants. Developmental Cognitive Neuroscience, 35, 20-27. doi: 10.1016/j.dcn.2017.10.008

Mireault, G. C., Rainville, B. S., \& Laughlin, B. (2018). Push or carry? Pragmatic opportunities for language development in strollers versus backpacks. Infancy, 23(4), 616-624. doi: 10.1111/infa.12238

Moreno, A. J., Posada, G. E., \& Goldyn, D. T. (2006). Presence and quality of touch influence coregulation in mother-infant dyads. Infancy, 9(1), 1-20. doi: 10.1207/s15327078in0901_1

Morrison, I. (2016). Keep calm and cuddle on: Social touch as a stress buffer. Adaptive Human Behavior and Physiology, 2, 344-362. doi: 10.1007/s40750-016-0052-x

Murray, L., De Pascalis, L., Bozicevic, L., Hawkins, L., Sclafani, V., \& Ferrari, P. F. (2016). The functional architecture of mother-infant communication, and the development of infant social expressiveness in the first two months. Scientific Reports, 6, 39019. doi: 10.1038/srep39019

Muschinski, J., Feczko, E., Brooks, J. M., Collantes, M., Heitz, T. R., \& Parr, L. A. (2016). The development of visual preferences for direct versus averted gaze faces in infant macaques (Macaca mulatta). Developmental Psychobiology, 58(8), 926-936. doi: 10.1002/dev.21421

Nagy, E. (2011). The newborn infant: A missing stage in developmental psychology. Infant and Child Development, 20(1), 3-19. doi: 10.1002/icd.683

Nagy, E., Pilling, K., Watt, R., Pal, A., \& Orvos, H. (2017). Neonates' responses to repeated exposure to a still face. PLOS ONE, 12(8), e01. doi: 10.1371/journal.pone.0181688

Nishizato, M., Fujisawa, T. X., Kosaka, H., \& Tomoda, A. (2017). Developmental changes in social attention and oxytocin levels in infants and children. Scientific Reports, 7(1), 2540. doi: 10.1038/s41598-017-02368-x

Olausson, H., Lamarre, Y., Backlund, H., Morin, C., Wallin, B. G., Starck, G., ... \& Bushnell, M. C. (2002). Unmyelinated tactile afferents signal touch and project to insular cortex. Nature Neuroscience, 5(9), 900-904. doi: 10.1038/nn896

Orchard-Mills, E., Alais, D., \& Van der Burg, E. (2013). Cross-modal associations between vision, touch, and audition influence visual search through top-down attention, not bottom-up capture. Attention, Perception, \& Psychophysics, 75(8), 1892-1905. doi: 10.3758/s13414-013-0535-9

Ordy, J. M., Latanick, A., Samorajski, T., \& Massopust, L. C. (1964). Visual acuity in newborn primate infants. Proceedings for the Society of Experimental Biology and Medicine, 115, 677-680. doi: 10.3181/00379727-115-29004

Peláez-Nogueras, M., Gewirtz, J. L., Field, T., Cigales, M., Malphurs, J., Clasky, S., \& Sanchez, A. (1996). Infants' preference for touch stimulation in face-to-face interactions. Journal of Applied Developmental Psychology, 17(2), 199-213. doi: 10.1016/S0193-3973(96)90025-8

Panagiotopoulou, E., Filippetti, M. L., Gentsch, A., \& Fotopoulou, A. (2018). Dissociable sources of erogeneity in social touch: Imagining and perceiving C-Tactile optimal touch in erogenous zones. PLOS ONE, 13(8), e0203039. doi: 10.1371/journal.pone.0203039

Parr, L. A., Brooks, J. M., Jonesteller, T., Moss, S., Jordano, J. O., \& Heitz, T. R. (2016). Effects of chronic oxytocin on attention to dynamic facial expressions in infant macaques. Psychoneuroendocrinology, 74, 149-157. doi: 10.1016/j.psyneuen.2016.08.028

Paukner, A., Simpson, E. A., Ferrari, P. F., Mrozek, T., \& Suomi, S. J. (2014). Neonatal imitation predicts how infants engage with faces. Developmental Science, 17(6), 833-840. doi: 10.1111/desc.12207

Paukner, A., Slonecker, E. M., Murphy, A. M., Wooddell, L. J., \& Dettmer, A. M. (2018). Sex and rank affect how infant rhesus macaques look at faces. Developmental Psychobiology, 60(2), 187-193. doi: 10.1002/dev.21579

Pawling, R., Cannon, P. R., McGlone, F. P., \& Walker, S. C. (2017). C-tactile afferent stimulating touch carries a positive affective value. PLOS ONE, 12(3), e0173457. doi:

10.1371/journal.pone.0173457 
Pierce, K., Marinero, S., Hazin, R., McKenna, B., Barnes, C. C., \& Malige, A. (2016). Eye tracking reveals abnormal visual preference for geometric images as an early biomarker of an autism spectrum disorder subtype associated with increased symptom severity. Biological Psychiatry, 79(8), 657666. doi: 10.1016/j.biopsych.2015.03.032

Pirazzoli, L., Lloyd-Fox, S., Braukmann, R., Johnson, M. H., \& Gliga, T. (2019). Hand or spoon? Exploring the neural basis of affective touch in 5-month-old infants. Developmental Cognitive Neuroscience, 35, 28-35. doi: 10.1016/j.dcn.2018.06.002

Reeb-Sutherland, B. C. (2018). What environmental factors contribute to the development of anxiety in temperamentally inhibited children? Insight from animal research models. Policy Insights from the Behavioral and Brain Sciences, 5(1), 126-133. doi: 10.1177/2372732217743990

Reece, C., Ebstein, R., Cheng, X., Ng, T., \& Schirmer, A. (2016). Maternal touch predicts social orienting in young children. Cognitive Development, 39, 128-140. doi: 10.1016/j.cogdev.2016.05.001

Ryan, A. M., Freeman, S. M., Murai, T., Lau, A. R., Palumbo, M. C., Hogrefe, C. E., Bales, K. L., \& Bauman, M. D. (2019). Non-invasive eye tracking methods for New World and Old World monkeys. Frontiers in Behavioral Neuroscience, 13, 39. doi: 10.3389/fnbeh.2019.00039

Schirmer, A., Ng, T., \& Ebstein, R. P. (2018). Vicarious social touch biases gazing at faces and facial emotions. Emotion, 18(8), 1097.

Schneider, M. L., Moore, C. F., Suomi, S. J., \& Champoux, M. (1991). Laboratory assessment of temperament and environmental enrichment in rhesus monkey infants (Macaca mulatta). American Journal of Primatology, 25(3), 137-155. doi: 10.1002/ajp.1350250302

Sclafani, V., Paukner, A., Suomi, S. J., \& Ferrari, P. F. (2015). Imitation promotes affiliation in infant macaques at risk for impaired social behaviors. Developmental Science, 18(4), 614-621. doi: $10.1111 /$ desc. 12237

Shepley, M. M. (2006). The role of positive distraction in neonatal intensive care unit settings. Journal of Perinatology, 26(S3), S34. doi: 10.1038/sj.jp.7211584

Shultz, S., Klin, A., \& Jones, W. (2018). Neonatal transitions in social behavior and their implications for autism. Trends in Cognitive Sciences, 22(5), 452-469. doi: 10.1016/j.tics.2018.02.012

Shibata, M., Fuchino, Y., Naoi, N., Kohno, S., Kawai, M., Okanoya, K., \& Myowa-Yamakoshi, M. (2012). Broad cortical activation in response to tactile stimulation in newborns. Neuroreport, 23(6), 373377. doi: 10.1097/WNR.0b013e3283520296

Simpson, E. A., Jakobsen, K. V., Damon, F., Suomi, S. J., Ferrari, P. F., \& Paukner, A. (2017). Face detection and the development of own-species bias in infant macaques. Child Development, 88(1), 103-113. doi: 10.1111/cdev.12565

Simpson, E. A., Miller, G. M., Ferrari, P. F., Suomi, S. J., \& Paukner, A. (2016a). Neonatal imitation and early social experience predict gaze following abilities in infant monkeys. Scientific Reports, 6, 20233. doi: 10.1038/srep20233

Simpson, E. A., Murray, L., Paukner, A., \& Ferrari, P. F. (2014). The mirror neuron system as revealed through neonatal imitation: Presence from birth, predictive power, and evidence of plasticity. Philosophical Transactions of the Royal Society B: Biological Sciences, 369(1644), 1-12. doi: 10.1098/rstb.2013.0289

Simpson, E. A., Nicolini, Y., Shetler, M., Suomi, S. J., Ferrari, P. F., \& Paukner, A. (2016b). Experienceindependent sex differences in newborn macaques: Females are more social than males. Scientific Reports, 6, 19669. doi: 10.1038/srep19669

Simpson, E. A., Paukner, A., Sclafani, V., Kaburu, S. S., Suomi, S. J., \& Ferrari, P. F. (2017). Acute oxytocin improves memory and gaze following in male but not female nursery-reared infant macaques. Psychopharmacology, 234(3), 497-506. doi: 10.1007/s00213-016-4480-x 
Simpson, E. A., Sclafani, V., Paukner, A., Hamel, A. F., Novak, M. A., Meyer, J. S., ... \& Ferrari, P. F. (2014b). Inhaled oxytocin increases positive social behaviors in newborn macaques. Proceedings of the National Academy of Sciences, 111(19), 6922-6927. doi: 10.1073/pnas.1402471111

Simpson, E. A., Sclafani, V., Paukner, A., Kaburu, S. S., Suomi, S. J., \& Ferrari, P. F. (2019). Handling newborn monkeys alters later exploratory, cognitive, and social behaviors. Developmental Cognitive Neuroscience, 35, 12-19. doi: 10.1016/j.dcn.2017.07.010

Simpson, E. A., Suomi, S. J., \& Paukner, A. (2016). Evolutionary relevance and experience contribute to face discrimination in infant macaques (Macaca mulatta). Journal of Cognition and Development, 17(2), 285-299. doi: 10.1080/15248372.2015.1048863

Spinelli, S., Schwandt, M. L., Lindell, S. G., Heilig, M., Suomi, S. J., Higley, J. D., ... \& Barr, C. S. (2012). The serotonin transporter gene linked polymorphic region is associated with the behavioral response to repeated stress exposure in infant rhesus macaques. Development and Psychopathology, 24(1), 157-165. doi: 10.1017/S0954579411000745

Stack, D. M., \& Muir, D. W. (1992). Adult tactile stimulation during face-to-face interactions modulates five-month-olds' affect and attention. Child Development, 63(6), 1509-1525. doi: 10.1111/j.1467-8624.1992.tb01711.x

Suvilehto, J. T., Glerean, E., Dunbar, R. I., Hari, R., \& Nummenmaa, L. (2015). Topography of social touching depends on emotional bonds between humans. Proceedings of the National Academy of Sciences, 112(45), 13811-13816. doi: 10.1073/pnas.1519231112

Taylor, S. E. (2006). Tend and befriend: Biobehavioral bases of affiliation under stress. Current Directions in Psychological Science, 15(6), 273-277. doi: 10.1111/j.1467-8721.2006.00451.x

Taylor, S. E., Klein, L. C., Lewis, B. P., Gruenewald, T. L., Gurung, R. A., \& Updegraff, J. A. (2000). Biobehavioral responses to stress in females: tend-and-befriend, not fight-or-flight. Psychological Review, 107(3), 411.

Underdown, A., Barlow, J., \& Stewart-Brown, S. (2010). Tactile stimulation in physically healthy infants: results of a systematic review. Journal of Reproductive and Infant Psychology, 28(1), 11-29. doi: 10.1080/02646830903247209

Vallbo, A. B., Olausson, H., Wessberg, J. (1999). Unmyelinated afferents constitute a second system coding tactile stimuli of the human hairy skin. Journal of Neurophysiology, 81, 2753-2763. doi: 10.1152/jn.1999.81.6.2753

Van Horen, F., \& Mussweiler, T. (2014). Soft assurance: Coping with uncertainty through haptic sensations. Journal of Experimental Social Psychology, 54, 73-80. doi: 10.1016/j.jesp.2014.04.008

Vanderwert, R. E., Simpson, E. A., Paukner, A., Suomi, S. J., Fox, N. A., \& Ferrari, P. F. (2015). Early social experience affects neural activity to affiliative facial gestures in newborn nonhuman primates. Developmental Neuroscience, 37(3), 243-252. doi: 10.1159/000381538

Watt, J. (1990). Interaction, intervention, and development in small-for-gestational-age infants. Infant Behavior and Development, 13(3), 273-286. doi: 10.1016/0163-6383(90)90035-7

Weaver, I. C., Cervoni, N., Champagne, F. A., D'Alessio, A. C., Sharma, S., Seckl, J. R., ... \& Meaney, M. J. (2004). Epigenetic programming by maternal behavior. Nature Neuroscience, 7(8), 847-854. doi: $10.1038 / \mathrm{nn} 1276$ 
Figures
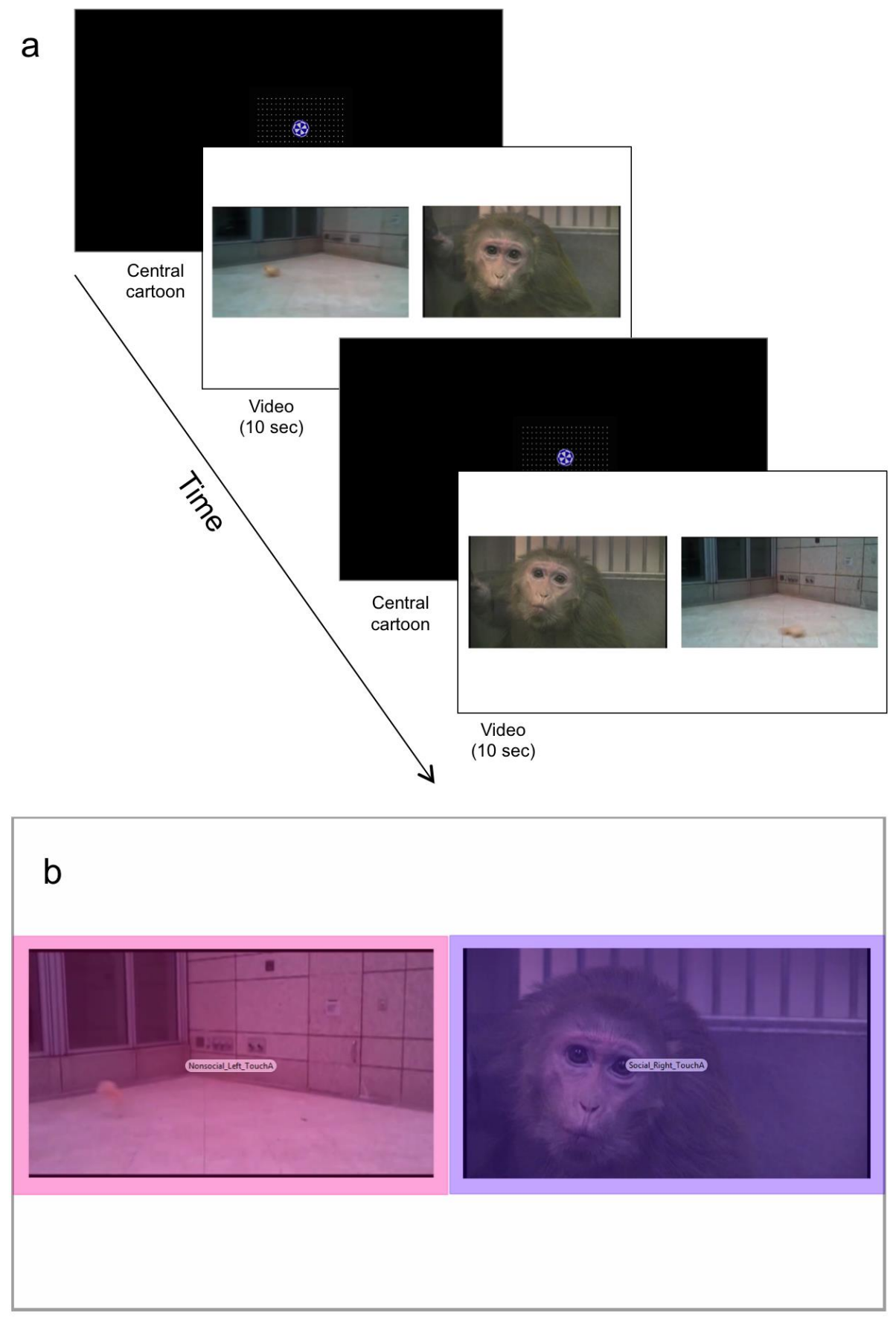

Figure 1. Sample (a) video stimulus presentation order, and (b) eye tracking areas of interest (AOIs). Nonsocial (plastic bag floating in the wind) and social (conspecific producing an affiliative/positive facial expression) video pairs played concurrently for 10 seconds, then the side of the social and nonsocial were switched for a second 10 second presentation, to control for potential side bias effects. 

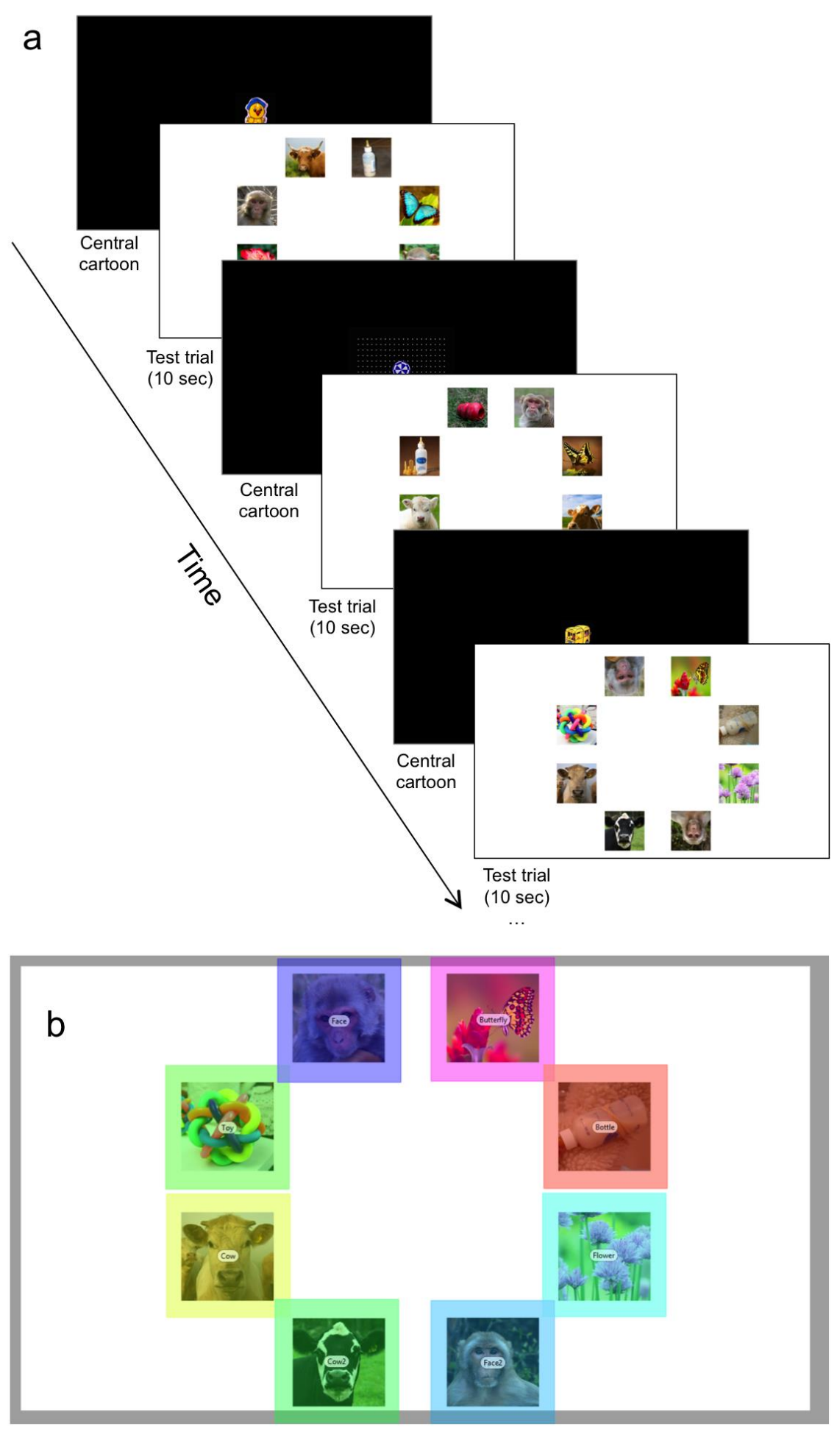

Figure 2. Picture array stimuli sample (a) stimulus presentation, and (b) eye tracking areas of interest (AOIs). 




Figure 3. During caregiver interactions, infants in the No Touch condition (light blue bar) displayed higher internalizing stress composite scores (scratching, time in contact with surrogate [a comfort object], and time moving around [locomotion]), compared to either of the touch groups (dark blue bars), ${ }^{*} p s<.05$, suggesting touch may reduce infants' stress levels. Error bars reflect standard error of the mean. 



Figure 4. Following caregiver interactions, infants' attention was impacted by condition (No Touch, Hand/Foot [non-CT touch], Head/Shoulder [CT-targeted touch]) (a) Infants looked a significantly greater proportion of time to the non-social relative to the social video in the No Touch condition (light red bar), but looked equally to the social and nonsocial videos (.5 = chance looking, represented by the dashed line) in both touch conditions (dark red bars), suggesting touch may shift infants to attend relatively less to dynamic but not socially relevant stimuli (instead attending more equally to dynamic socially relevant and nonsocial stimuli). (b) Infants were significantly faster to look to the image array in the Head/Shoulder Touch condition (far right dark purple bar) compared to the both the No Touch and the Hand/Foot Touch conditions (left two purple bars), suggesting CT-targeted touch may facilitate infants' attention capture. Notably, this later effect was not specific to social stimuli (face photos), but was a more general effect for orienting to all photo stimuli (social and non-social). Error bars reflect standard error of the mean, $* p s \leq .054$. 


\begin{tabular}{lll}
\hline Type & Behavior & Operational Definition \\
\hline Events & LPS & Lipsmacking: Rapid opening and closing of the mouth \\
States & Vis attn & Visual attention: Looking at the face of the human caregiver model \\
& Prox & Close proximity to caregiver: Torso within $5 \mathrm{~cm}$ (arm's reach) from cage front \\
Events & Scratch & Common use \\
& Yawn & Common use \\
& Spasm & Sudden jerking movement made by infant \\
& Voc & Vocalization made by infant (any type) \\
States & Self-suck & Insertion of fingers/toes into mouth \\
& Self-clasp & Hand or foot closed on any body part \\
& Surrogate & Touching of surrogate \\
& Loco & Locomotion: Directed movement of torso ( $>15 \mathrm{~cm}$ within $5 \mathrm{sec}$ ) \\
& Explore & Exploration: Manipulating toys or bedding with hands, feet, or mouth \\
& Sleep & Infant lying down and not moving \\
\hline
\end{tabular}

Table 1. Behaviors coded during the touch manipulation sessions. Scored behaviors included events (frequency counts) and states (time durations in seconds). Coders were blind to the study hypotheses, but could see the infant being touched in the videos. 


\section{Infant Behavior During Caregiver Interactions}

During caregiver interactions, there were effects of condition for only two behaviors: locomotion and exploration, Figure S1c-d (see main paper for details). While not reaching the Bonferroni-adjusted levels of statistical significance $(p<.005)$, we did find additional trends $(p s<.05)$, described below.

We found a trend of an effect of condition on scratching, $F(2,52)=3.204, p=.049, \eta_{p}^{2}=.110$, in which infants scratched less in the Hand/Foot condition $(M=.85, S D=1.06)$ than in the No Touch condition $(M=2.04, S D=2.75), t(26)=2.27, p=.032, d=0.44$, Figure $S 1 a$. This may indicate that infants were more stressed in the no-touch condition compared to the Hand/Foot condition, consistent with the proposal that social touch may reduce infants' stress. However, we detected no significant differences in scratching between the Head/Shoulder $(M=1.07, S D=1.75)$ and Hand/Foot conditions $(M=.85, S D=1.06), t(26)=.78, p=.44$, nor between the Head/Shoulder and No Touch conditions $(M=$ $2.04, S D=2.75), t(26)=1.54, p=.032$. The effects of touch on scratching may therefore be small.

There was a trend of an effect of condition on time spent in contact with the surrogate, $F(2,52)$ $=3.750, p=.030, \eta_{p}{ }^{2}=.126$, Figure S1b. Infants touched the surrogate less in the Head/Shoulder $(M=$ 45.94 seconds, $S D=79.25)$ compared to the No Touch condition $(M=88.69$ seconds, $S D=73.99), t(26)=$ 2.27, $p=.032, d=0.44$, and touched the surrogate more in the No Touch compared to the Hand/Foot condition $(M=48.41$ seconds, $S D=54.44), t(26)=2.51, p=.018, d=0.49$. There was no difference in touching the surrogate between the Head/Shoulder and Hand/Foot conditions, $t(26)=.14, p=.89$. These findings suggest that infants may seek out contact comfort from their surrogate when a caregiver does not already meet that need.

We also found an effect of condition on spasm rates, $F(2,52)=3.67, p=.032, \eta_{p}{ }^{2}=.124$, Figure S1g, in which infants spasmed more in the Head/Shoulder $(M=3.78, S D=7.47)$ than the No Touch condition $(M=.85, S D=2.28), t(26)=2.17, p=.039, d=0.43$, Figure S1g. There were no differences in spasms between the Hand/Foot $(M=2.93, S D=5.43)$ and No Touch conditions, $t(26)=1.96, p=.06$, nor between the Head/Shoulder and Hand/Foot conditions, $t(26)=.97, p=.34$. Spasms may be a way for infants to communicate with caretakers that they are uncomfortable, which occurred more often when in direct physical contact.

There was also an effect of condition on visual attention to the human caretaker, $F(2,52)=4.88$, $p=.011, \eta_{p}{ }^{2}=.158$, Figure S1e. Infants attended more in the Head/Shoulder $(M=29.16$ seconds, $S D=$ $35.28)$ than in the Hand/Foot condition ( $M=13.78$ seconds, $S D=10.91), t(26)=2.10, p=.046, d=0.41$. Infants attended more in the No Touch $(M=34.59, S D=28.81)$ than in the Hand/Foot condition, $t(26)=$ $3.40, p=.002, d=.67$. There was no difference in attention between the No Touch and Head/Shoulder conditions, $t(26)=.75, p=.46$. Infants in the Hand/Foot condition may have been attending to their body part being touched, which was in view. In contrast, in the other conditions, the body parts touched were outside of view (Head/Shoulder) or they were not being touched, so in these cases infants may have been seeking out face-to-face interactions with their caregivers more frequently.

There were no significant condition effects on lipsmacking, $F(2,52)=2.37, p=.10$, Figure $\mathrm{S} 1 \mathrm{i}$, time in proximity, $F(2,52)=.96, p=.39$, Figure S1f, or yawning, $F(2,52)=.15, p=.86$, Figure S1h. 

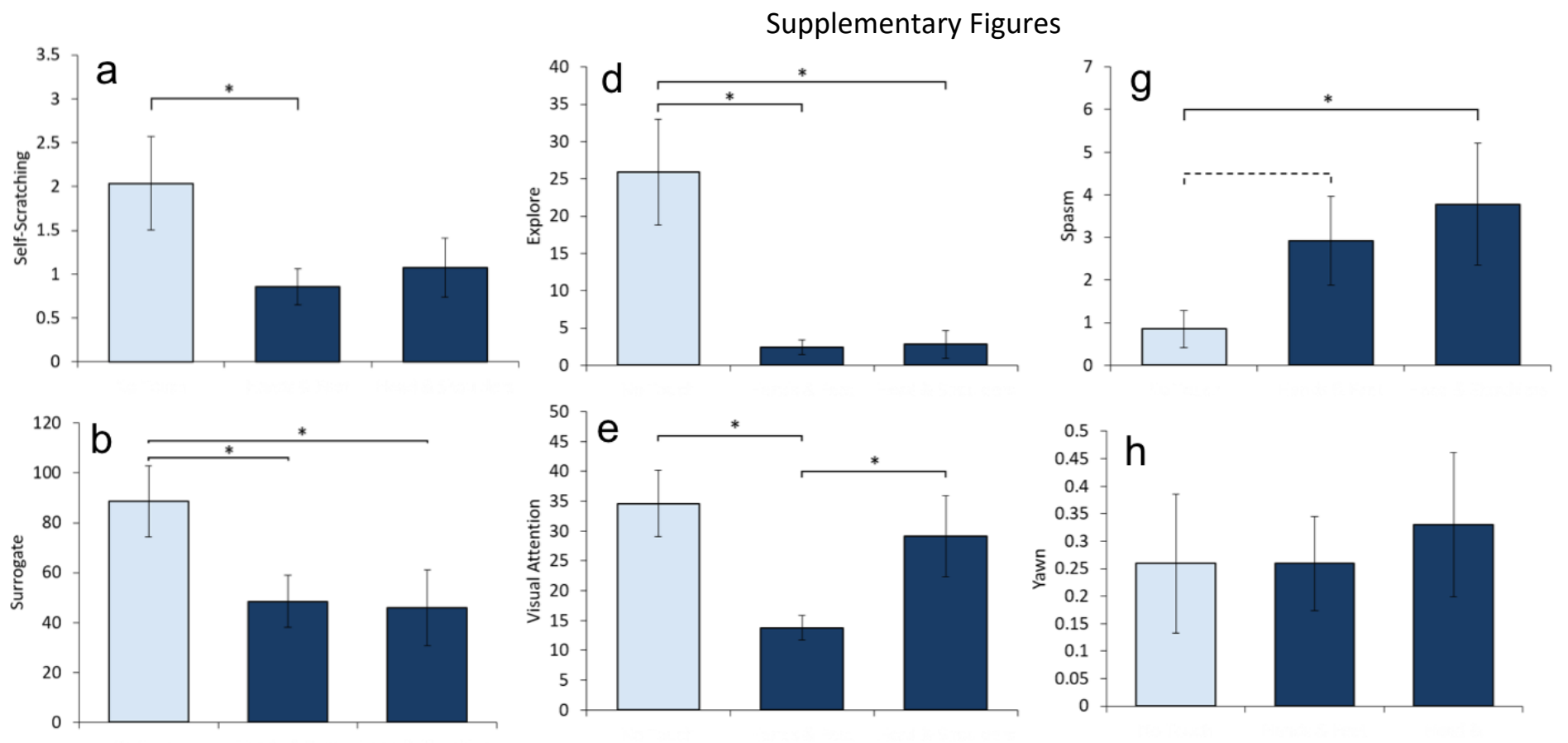

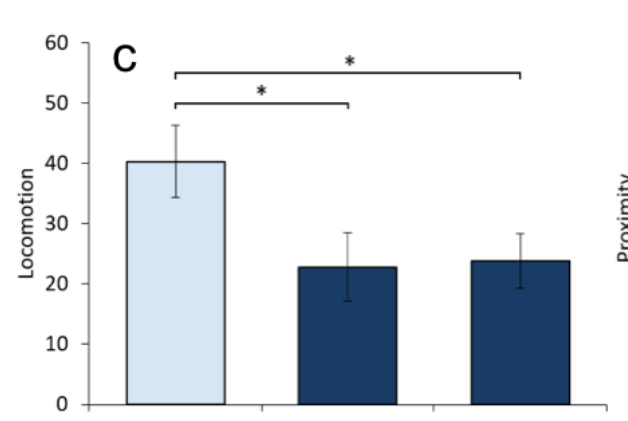

No Touch Hand/Foot Head/Shoulder
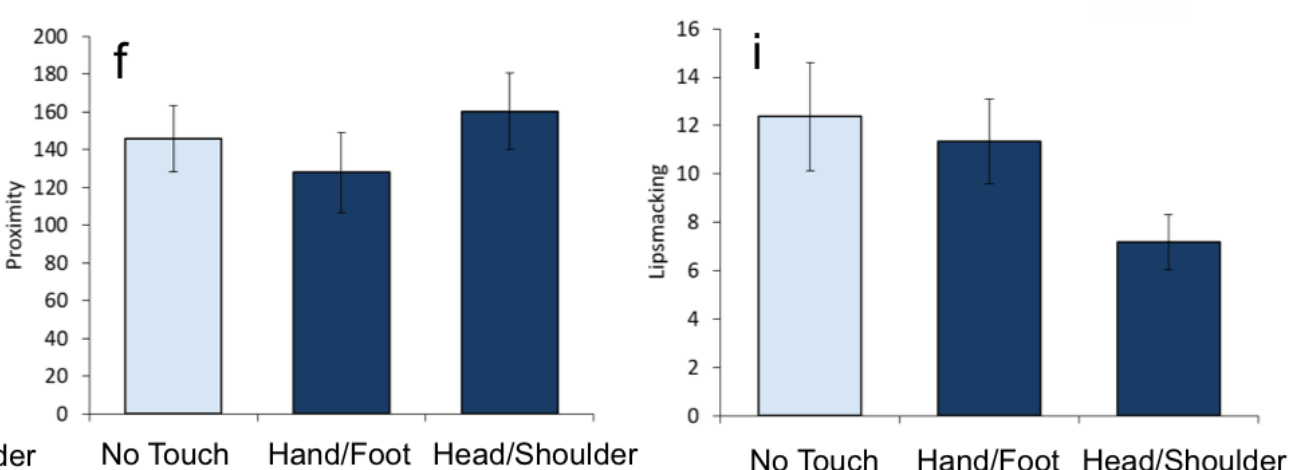

Figure S1. During the caregiver interactions, infants in the No Touch condition (light blue), compared to the touch conditions (dark blue), displayed higher rates of (a) self-scratching (rate per minute), (b) time in contact with the surrogate (sec), (c) time spent in locomotion (sec), (d) time spent exploring (sec). (d) Infants in the CT-targeted touch (Hand/Foot) condition, were less attentive to the caregiver (sec) compared to the other conditions, but did not differ in (f) proximity to the caregiver (sec). (g) Infants in the No Touch condition spasmed less than the other conditions. There were no effects for (h) yawning or (i) lipsmacking. Error bars reflect standard error of the mean, * $p s<.05$. 


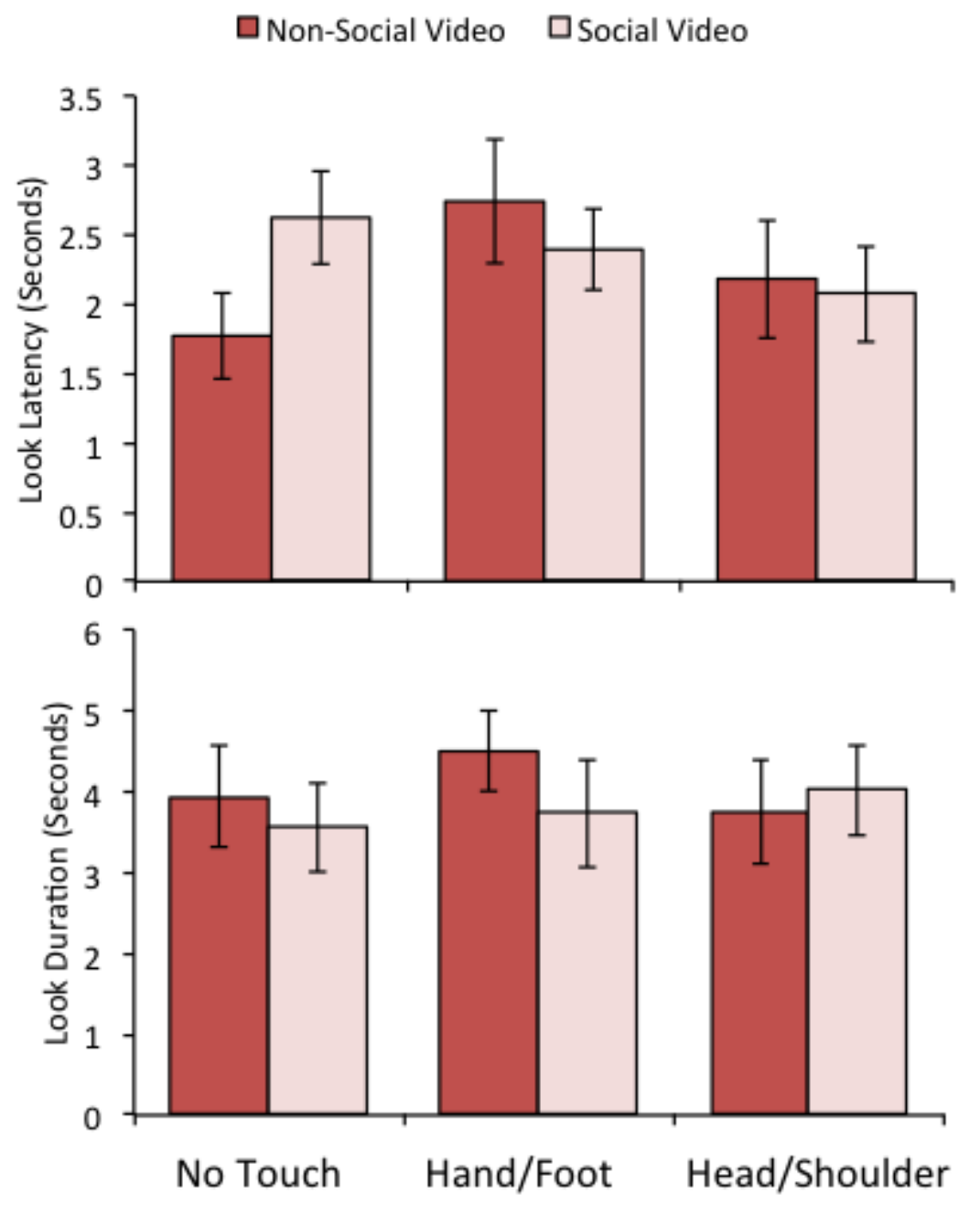

Figure S2. Following caregiver interactions, we found no statistically significant differences in condition (No Touch, Hand/Foot, Head/Shoulder) for infants' attention to the nonsocial (dark red) and social (light red) videos for either look latency (top graph) or duration (bottom graph), ps > .05. 
$\square$ Non-Social Pictures $\quad \square$ Social Pictures


Figure S3. Following caregiver interactions, we found no statistically significant differences in condition (No Touch, Hand/Foot, Head/Shoulder) for infants' attention to the nonsocial (dark purple) and social (light purple) photos for either look latency (top graph) or duration (bottom graph), ps > .05. Social photos included face photos of conspecifics. Nonsocial photos included photos of familiar and novel objects. 
Supplementary Tables

\begin{tabular}{|c|c|c|c|c|c|c|c|c|c|c|}
\hline & & 1 & 2 & 3 & 4 & 5 & 6 & 7 & 8 & 9 \\
\hline 1 & LPS & -- & & & & & & & & \\
\hline 2 & Vis Attn & $0.282^{*}$ & -- & & & & & & & \\
\hline 3 & Prox & 0.007 & $0.292 * *$ & -- & & & & & & \\
\hline 4 & Spasm & -0.048 & 0.117 & 0.201 & -- & & & & & \\
\hline 5 & Yawn & $0.322 * *$ & 0.101 & $-0.226^{*}$ & 0.004 & -- & & & & \\
\hline 6 & Voc & -0.053 & $0.281^{*}$ & 0.156 & $0.294 * *$ & $0.257^{*}$ & -- & & & \\
\hline 7 & Scratch & 0.187 & 0.052 & -0.215 & $-0.240^{*}$ & 0.213 & -0.149 & -- & & \\
\hline 8 & Surrogate & -0.023 & -0.045 & $-0.533^{* *}$ & -0.209 & -0.018 & -0.129 & $0.279 *$ & -- & \\
\hline 9 & Loco & -0.029 & -0.208 & -0.178 & -0.125 & -0.110 & -0.200 & -0.050 & $0.220 *$ & -- \\
\hline
\end{tabular}

Table S1. Associations among behaviors exhibited by infants during the 5-minute caregiver interaction with mutual gaze time period (with No Touch, Hand/Foot Touch, or Head/Shoulder Touch), including lipsmacking (LPS), visual attention to the caregiver (Vis Attn), time in close proximity to the caregiver (Prox), spasms, yawns, vocalizations (Voc), scratching, time in contact with the surrogate, and locomotion (Loco); ${ }^{*} p<.05,{ }^{* *} p<.01$. 